\title{
AMINO ACID DERIVED ENAMINONES: A STUDY IN RING FORMATION PROVIDING VALUABLE ASYMMETRIC SYNTHONS
}

\author{
Brandon J. Turunen and Gunda I. Georg* \\ Department of Medicinal Chemistry and Center for Methodology and Library \\ Development, University of Kansas, 1251 Wescoe Hall, Drive Lawrence, Kansas 66045
}

1. General experimental paragraph...................................... 2

2. Representative procedures and characterization data for 2 and $3 \ldots \ldots \ldots$. 2 2-S5

3. Characterization data for compounds 4-26, 29 and 30.................S5-S17

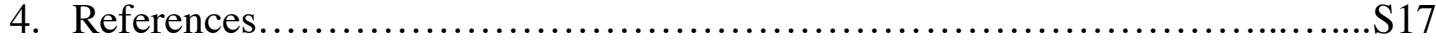

5. ${ }^{1} \mathrm{H}$ and ${ }^{13} \mathrm{C}$ spectra for compounds $2,4-19,21-26,29$, and 30.............S18-S69 


\section{General experimental paragraph.}

All commercially available reagents and solvents were used without further purification unless otherwise noted. Methylene chloride was dried by distillation from calcium hydride. Flash column chromatography was carried out on silica gel. TLC was conducted on silica gel 250 micron, $F_{254}$ plates. ${ }^{1} \mathrm{H}$ NMR spectra were recorded on 400 $\mathrm{MHz}$ or $500 \mathrm{MHz}$ NMR instruments. Chemical shifts are reported in ppm with the solvent as internal standard $\left(\mathrm{CDCl}_{3}: 7.28 \mathrm{ppm}\right)$. Data are reported as follows: chemical shift, integration, multiplicity $(\mathrm{s}=$ singlet, $\mathrm{d}=$ doublet, $\mathrm{t}=$ triplet, $\mathrm{q}=$ quartet, $\mathrm{br}=$ broad, $\mathrm{m}=$ multiplet), coupling constants $(\mathrm{Hz})$ and assignment. ${ }^{13} \mathrm{C}$ NMR spectra were recorded on $100 \mathrm{MHz}$ or $125 \mathrm{MHz}$ NMR spectrometers with complete proton decoupling. Chemical shifts are reported in ppm with the solvent as internal standard $\left(\mathrm{CDCl}_{3}: 77.0\right.$ ppm). High-resolution mass spectrometry was performed by the University of Kansas Mass Spectroscopy Service Laboratory. All new compounds were determined to be $>95 \%$ as determined by ${ }^{1} \mathrm{H}$ NMR spectroscopy.

\section{Representative procedure for N-Boc ynone preparation.}

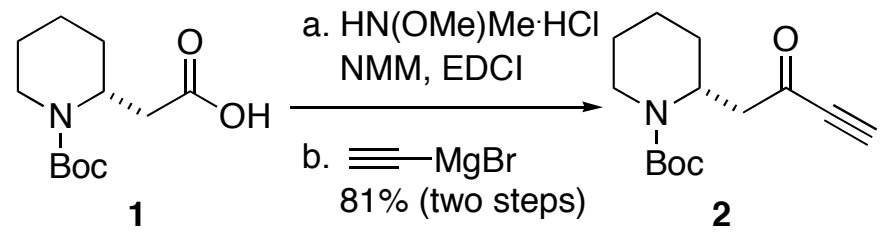

(S)-tert-Butyl 2-(2-(Methoxy(methyl)amino)-2-oxoethyl)piperidine-1-carboxylate (1a). (R)-2-(1-(tert-Butoxycarbonyl)piperidin-2-yl)acetic acid (1, $1.00 \mathrm{~g}, 4.0 \mathrm{mmol})$ was dissolved in anhydrous $\mathrm{CH}_{2} \mathrm{Cl}_{2}(100 \mathrm{~mL})$ under argon atmosphere and cooled to $-15{ }^{\circ} \mathrm{C}$. To this solution was added $\mathrm{N}, \mathrm{O}$-dimethylhydroxylamine $\mathrm{HCl}(0.42 \mathrm{~g}, 4.27 \mathrm{mmol})$ and $\mathrm{N}$ - 
methylmorpholine $(0.05 \mathrm{~mL}, 4.44 \mathrm{mmol})$ followed by EDCI $(0.82 \mathrm{~g}, 4.27 \mathrm{mmol})$ in portions over 30 minutes then allowed to come to room temperature. After 2 hours the reaction was again cooled to $0{ }^{\circ} \mathrm{C}$ and quenched by the addition of an ice cold $10 \% \mathrm{HCl}$ solution $(25 \mathrm{~mL})$ and allowed to stir at this temperature for 5 minutes. The reaction was diluted with water $(50 \mathrm{~mL})$ and extracted with $\mathrm{CH}_{2} \mathrm{Cl}_{2}(\mathrm{x} 3)$. The combined organic layers were washed with saturated $\mathrm{NaHCO}_{3}(\mathrm{x} 1)$, dried over $\mathrm{Na}_{2} \mathrm{SO}_{4}$, filtered and concentrated. This crude material was purified via flash chromatography (3 hexane/1 EtOAc) to provide $1.14 \mathrm{~g}$ of the amide as an oily solid (96\%): ${ }^{1} \mathrm{H} \mathrm{NMR}\left(400 \mathrm{MHz}, \mathrm{CDCl}_{3}, 50{ }^{\circ} \mathrm{C}\right) \square$ $1.33-1.45(\mathrm{bm}, 1 \mathrm{H}) 1.43(\mathrm{~s}, 9 \mathrm{H}), 1.44-1.52(\mathrm{bm}, 5 \mathrm{H}), 2.47-2.59(\mathrm{~m}, 2 \mathrm{H}), 2.70(\mathrm{bt}, J=13$ $\mathrm{Hz}, 1 \mathrm{H}), 3.01(\mathrm{~s}, 3 \mathrm{H}), 3.57(\mathrm{~s}, 3 \mathrm{H}), 3.88(\mathrm{bd}, J=13 \mathrm{~Hz}, 1 \mathrm{H}), 4.58(\mathrm{bs}, 1 \mathrm{H}) ;{ }^{13} \mathrm{C} \mathrm{NMR}$ $\left(100 \mathrm{MHz}, \mathrm{CDCl}_{3}, 50{ }^{\circ} \mathrm{C}\right) \square 19.3,25.7,28.8,32.4,33.1,39.7,48.0,61.6,79.7,155.1$, 172.6; IR (neat) 3564, 2933, 1690, 1447, $1255 \mathrm{~cm}^{-1}$; HRMS (ES+) $\mathrm{m} / z$ calc'd for $[\mathrm{M}+\mathrm{H}]^{+}$ $\mathrm{C}_{14} \mathrm{H}_{27} \mathrm{~N}_{2} \mathrm{O}_{4}: 287.1971$, found 287.1957; [ $]^{22}{ }_{D}-6.0\left(c=1.0, \mathrm{CHCl}_{3}\right)$.

(R)-tert-Butyl 2-(2-Oxobut-3-ynyl)piperidine-1-carboxylate (2). The Weinreb amide (1a, $0.72 \mathrm{~g}, 2.52 \mathrm{mmol})$ was dissolved in anhydrous THF (40 mL) under argon atmosphere and cooled to $0{ }^{\circ} \mathrm{C}$. To this reaction vessel, was added dropwise, a $0.5 \mathrm{M}$ solution of ethynyl magnesium bromide $(12.6 \mathrm{~mL}, 6.3 \mathrm{mmol})$ in THF and allowed to come to room temperature. After 2 hours the reaction was judged complete by TLC (1 hexanes / 1 EtOAc) and again cooled to $0{ }^{\circ} \mathrm{C}$. The reaction was quenched by the addition of an ice cold $10 \% \mathrm{HCl}$ solution $(15 \mathrm{~mL})$ and allowed to stir at this temperature for 5 minutes. The reaction was diluted with water and extracted with EtOAc (x3). The combined organic layers were washed with saturated $\mathrm{NaHCO}_{3}(\mathrm{x} 1)$, dried over $\mathrm{Na}_{2} \mathrm{SO}_{4}$, 
filtered and concentrated. This crude material was purified via flash chromatography (8 hexane/1 EtOAc) to provide $0.53 \mathrm{~g}$ of a colorless oil $(84 \%)$ : ${ }^{1} \mathrm{H}$ NMR $\left(500 \mathrm{MHz}, \mathrm{CDCl}_{3}\right)$ $1.41-1.51(\mathrm{~m}, 2 \mathrm{H}), 1.44(\mathrm{~s}, 9 \mathrm{H}), 1.59-1.76(\mathrm{~m}, 4 \mathrm{H}), 2.77-2.84(\mathrm{~m}, 2 \mathrm{H}), 2.87-2.90(\mathrm{~m}$, 1H), $3.29(\mathrm{~s}, 1 \mathrm{H}), 4.01(\mathrm{bs}, 1 \mathrm{H}), 4.85(\mathrm{bs}, 1 \mathrm{H}) ;{ }^{13} \mathrm{C} \mathrm{NMR}\left(125 \mathrm{MHz}, \mathrm{CDCl}_{3}\right) \square 18.8$, $25.1,28.3,28.4,39.1,45.8,47.4,78.8,79.8,81.5,154.5,185.0$; IR (neat) 3211,2937 , 2090, 1680, 1411, $1165 \mathrm{~cm}^{-1}$; HRMS (FAB+) $\mathrm{m} / \mathrm{z}$ calc'd for $[\mathrm{M}+\mathrm{Na}]^{+} \mathrm{C}_{14} \mathrm{H}_{21} \mathrm{NO}_{3} \mathrm{Na}$ : 274.1419, found 274.1419; [व] ${ }_{\mathrm{D}}^{22}-38\left(c=0.65, \mathrm{CHCl}_{3}\right)$.

\section{Representative procedure for conversion of ynone to enaminone:}<smiles>C#CC(=O)C[C@@H]1CCCCN1C(=O)OCc1ccccc1</smiles>

2

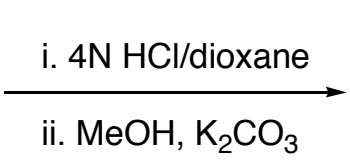

ii. $\mathrm{MeOH}, \mathrm{K}_{2} \mathrm{CO}_{3}$

$(R)$-7,8,9,9a-Tetrahydro-1 $H$-quinolizin-2(6H)-one (3). METHOD 1: Ynone (2, 72 $\mathrm{mg}, 0.48 \mathrm{mmol})$ was dissolved in a $4 \mathrm{~N} \mathrm{HCl}$-dioxane solution $(1.5 \mathrm{~mL})$ and allowed to react for 15 minutes. After this time the dioxane and excess $\mathrm{HCl}$ were removed under reduced pressure and this residue placed under vacuum for 15 minutes. This material was then dissolved in $\mathrm{MeOH}(10 \mathrm{~mL})$ and excess $\mathrm{K}_{2} \mathrm{CO}_{3}$ (a minimum of 4 equivalents) was added. The reaction was judged to be complete by TLC $\left(10 \% \mathrm{MeOH} / \mathrm{CH}_{2} \mathrm{Cl}_{2}\right)$ in 15 minutes. At this time $\mathrm{CH}_{2} \mathrm{Cl}_{2}$ was added, the reaction suction filtered, and the organic solvents concentrated. This residue was purified via flash chromatography (1-5\% $\left.\mathrm{MeOH} / \mathrm{CH}_{2} \mathrm{Cl}_{2}\right)$ to provide $37 \mathrm{mg}(87 \%)$ of an off-white solid: Spectral data of the title compound was identical to that reported in the literature with the exception of optical rotation: $[\square]_{D}^{22}-135\left(c=0.925, \mathrm{CHCl}_{3}\right)$. Comparison to the reported value, $[\square]_{\mathrm{D}}^{22}-146(c$ 
$\left.=0.885, \mathrm{CHCl}_{3}\right)$, indicated an ee of $93 \%$. This enantiomeric ratio was verified via chiral HPLC using a Baker Chiralcel OJ column. Conditions: isopropanol 2-15\% in hexanes, 60 minutes, $0.5 \mathrm{~mL} / \mathrm{min}, 30{ }^{\circ} \mathrm{C} .(-)$-Enantiomer: $\mathrm{R}_{\mathrm{t}}=40.1 \mathrm{~min} ; \quad(+)$-Enantiomer: $\mathrm{R}_{\mathrm{t}}=$ 41.6 min. ee $=94 \% .^{1}$

METHOD 2: The ynone $2(28 \mathrm{mg}, 0.11 \mathrm{mmol})$ was dissolved in anhydrous $\mathrm{CH}_{2} \mathrm{Cl}_{2}(10$ $\mathrm{mL})$ under an argon atmosphere and cooled to $-78{ }^{\circ} \mathrm{C}$. A solution of TMS-I $(0.03 \mathrm{~mL}$, $0.11 \mathrm{mmol})$ in anhydrous $\mathrm{CH}_{2} \mathrm{Cl}_{2}(1 \mathrm{~mL})$ was then added dropwise at this temperature. After 20 minutes at this temperature the reaction was allowed to warm to $0{ }^{\circ} \mathrm{C}$ and additional TMS-I (0.03-0.11 mmol) was added until all starting material was consumed (TLC, 3 hexanes / 1 EtOAc). After 20 minutes the reaction was judged complete and this mixture was concentrated under reduced pressure and placed under vacuum for 15 minutes. This residue was then dissolved in $\mathrm{MeOH}(5 \mathrm{~mL})$ and excess $\mathrm{K}_{2} \mathrm{CO}_{3}$ was added. The reaction was monitored via TLC $\left(10 \% \mathrm{MeOH} / \mathrm{CH}_{2} \mathrm{Cl}_{2}\right)$ and judged complete after 30 minutes. At this time $\mathrm{CH}_{2} \mathrm{Cl}_{2}$ was added and the resultant slurry suction filtered the organic layer concentrated. This residue was purified via flash chromatography (5\% $\left.\mathrm{MeOH} / \mathrm{CH}_{2} \mathrm{Cl}_{2}\right)$ to provide $16 \mathrm{mg}(95 \%)$ enaminone 3. The enantiomeric excess using this method was not determined on this example.

\section{Ynone characterization data (4-14):}

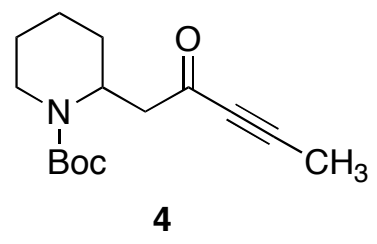


tert-Butyl 2-(2-Oxopent-3-ynyl)piperidine-1-carboxylate (4). ${ }^{1} \mathrm{H}$ NMR $(500 \mathrm{MHz}$, $\left.\mathrm{CDCl}_{3}\right) \square 1.25-1.48(\mathrm{~m}, 2 \mathrm{H}), 1.42(\mathrm{~s}, 9 \mathrm{H}), 1.55-1.67$ (m, 4H), $2.02(\mathrm{~s}, 3 \mathrm{H}), 2.75-2.80(\mathrm{~m}$, 3H), 4.00 (bs, $1 \mathrm{H}), 4.81$ (bs, $1 \mathrm{H}) ;{ }^{13} \mathrm{C}$ NMR $\left(125 \mathrm{MHz}, \mathrm{CDCl}_{3}\right) \square$ 4.1, 18.8, 25.2, 28.3, 39.2, 45.7, 47.6, 79.6, 80.3, 90.5, 154.6, 185.7; IR (neat) 2974, 2936, 2862, 2218, 1670, 1410, $1157 \mathrm{~cm}^{-1}$; HRMS (ES+) $\mathrm{m} / z$ calc'd for $[\mathrm{M}+\mathrm{H}]^{+} \mathrm{C}_{15} \mathrm{H}_{24} \mathrm{NO}_{3}: 266.1756$, found 266.1750.

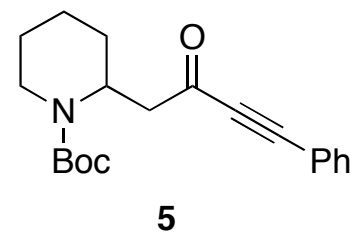

tert-Butyl 2-(2-Oxo-4-phenylbut-3-ynyl)piperidine-1-carboxylate (5). ${ }^{1} \mathrm{H}$ NMR (500 $\left.\mathrm{MHz}, \mathrm{CDCl}_{3}\right) \square 1.39-1.55(\mathrm{~m}, 2 \mathrm{H}), 1.44(\mathrm{~s}, 9 \mathrm{H}), 1.64-1.73(\mathrm{~m}, 4 \mathrm{H}), 2.85(\mathrm{dd}, J=14 \mathrm{~Hz}$, $9 \mathrm{~Hz}, 2 \mathrm{H}), 2.97$ (dd, $J=14 \mathrm{~Hz}, 7 \mathrm{~Hz}, 1 \mathrm{H}), 4.06$ (bs, 1H), 4.89 (bs, 1H), 7.23-7.41 (m, $2 \mathrm{H}), 7.44-7.47(\mathrm{~m}, 1 \mathrm{H}), 7.58(\mathrm{~d}, J=7 \mathrm{~Hz}, 2 \mathrm{H}) ;{ }^{13} \mathrm{C} \mathrm{NMR}\left(125 \mathrm{MHz}, \mathrm{CDCl}_{3}\right) \square$ 18.7, $25.2,28.3,39.1,45.8,47.8,53.4,79.6,88.0,91.0,119.8,128.5,130.6,133.0,154.5$, 185.6; IR (neat) $3410,2359,2202,1654 \mathrm{~cm}^{-1}$; HRMS (ES+) $\mathrm{m} / z$ calc'd for $[\mathrm{M}+\mathrm{H}]^{+}$ $\mathrm{C}_{20} \mathrm{H}_{26} \mathrm{NO}_{3}: 328.1913$, found 328.1907.

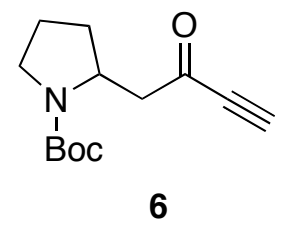

tert-Butyl 2-(2-Oxobut-3-ynyl)pyrrolidine-1-carboxylate (6). ${ }^{1} \mathrm{H}$ NMR (500 MHz, $\left.\mathrm{CDCl}_{3}, 50{ }^{\circ} \mathrm{C}\right) \square 1.38(\mathrm{~s}, 9 \mathrm{H}), 1.60-1.67(\mathrm{bs}, 1 \mathrm{H}), 1.72-1.80(\mathrm{~m}, 2 \mathrm{H}), 2,00-2.09(\mathrm{~m}, 1 \mathrm{H})$, 
2.52-2.59 (m, 1H), $3.19(\mathrm{~s}, 1 \mathrm{H}), 3.22(\mathrm{bm}, 3 \mathrm{H}), 4.13-4.22(\mathrm{bm}, 1 \mathrm{H}) ;{ }^{13} \mathrm{C}$ NMR $(125$ $\left.\mathrm{MHz}, \mathrm{CDCl}_{3}\right) \square 23.6,28.4,31.5,46.8,50.0,53.4,78.0,79.5,81.7,154.5,183.0$; IR (neat) 3209, 2977, 2881, 2092, 1707, 1414, $1179 \mathrm{~cm}^{-1}$; HRMS (FAB+) $\mathrm{m} / z$ calc'd for $[\mathrm{M}+\mathrm{H}]^{+}$ $\mathrm{C}_{13} \mathrm{H}_{20} \mathrm{NO}_{3}: 238.1443$, found 238.1440.

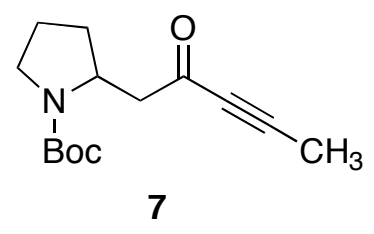

tert-Butyl 2-(2-Oxopent-3-ynyl)pyrrolidine-1-carboxylate (7). ${ }^{1} \mathrm{H}$ NMR (500 MHz, $\left.\mathrm{CDCl}_{3}, 50{ }^{\circ} \mathrm{C}\right) \square 1.43(\mathrm{~s}, 9 \mathrm{H}), 1.64(\mathrm{bs}, 1 \mathrm{H}), 1.78-1.99(\mathrm{~m}, 2 \mathrm{H}), 1.99$ (s, 3H), 2.00-2.08 $(\mathrm{m}, 1 \mathrm{H}), 2.45-2.53(\mathrm{~m}, 1 \mathrm{H}) 2.82-3.10(\mathrm{bs}, 1 \mathrm{H}), 3.28-3.40(\mathrm{~m}, 2 \mathrm{H}), 4.20(\mathrm{bs}, 1 \mathrm{H}) ;{ }^{13} \mathrm{C}$ NMR (125 MHz, $\left.\mathrm{CDCl}_{3}\right) \square 3.8,23.5,28.4,31.0,46.6,50.1,53.6,80.0,80.5,91.0,154.5$, 186.6; IR (neat) 2975, 2939, 2216, 1670, 1397, $1163 \mathrm{~cm}^{-1}$; HRMS (ES+) $\mathrm{m} / \mathrm{z}$ calc'd for $[\mathrm{M}+\mathrm{H}]^{+} \mathrm{C}_{14} \mathrm{H}_{22} \mathrm{NO}_{3}: 252.1600$, found 252.1591 .

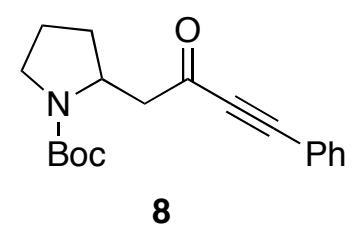

tert-Butyl 2-(2-Oxo-4-phenylbut-3-ynyl)pyrrolidine-1-carboxylate (8). ${ }^{1} \mathrm{H}$ NMR (500 $\left.\mathrm{MHz}, \mathrm{CDCl}_{3}, 50{ }^{\circ} \mathrm{C}\right) \mathrm{Q} 1.40(\mathrm{~s}, 9 \mathrm{H}), 1.60-1.66(\mathrm{bs}, 1 \mathrm{H}) 1.66-1.75(\mathrm{~m}, 2 \mathrm{H}), 1.96-2.02(\mathrm{~m}$, $1 \mathrm{H}), 2.55-2.61(\mathrm{~m}, 1 \mathrm{H}), 3.00-3.12(\mathrm{bs}, 1 \mathrm{H}), 3.19-3.29$ (bs, $2 \mathrm{H}), 4.12-4.18(\mathrm{~m}, 1 \mathrm{H}), 7.18-$ $7.21(\mathrm{~m}, 2 \mathrm{H}), 7.25-7.28(\mathrm{~m}, 1 \mathrm{H}), 7.38-7.42(\mathrm{~m}, 2 \mathrm{H}) ;{ }^{13} \mathrm{C} \mathrm{NMR}\left(125 \mathrm{MHz}, \mathrm{CDCl}_{3}\right)$ $23.5,28.6,31.4,36.7,50.4,54.0,56.8,80.0,88.2,91.3,120.1,128.5,130.6,133.0,154.4$, 
186.2; IR (neat) 2974, 2878, 2203, 1695, 1395, $1168 \mathrm{~cm}^{-1}$; HRMS (ES+) $\mathrm{m} / z$ calc'd for $[\mathrm{M}+\mathrm{H}]^{+} \mathrm{C}_{19} \mathrm{H}_{24} \mathrm{NO}_{3}: 314.1756$, found 314.1729.

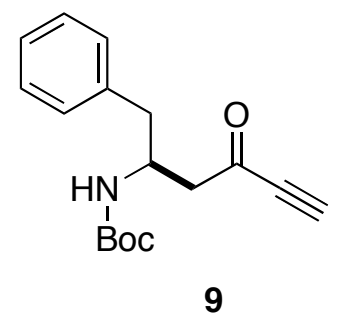

(S)-tert-Butyl 4-Oxo-1-phenylhex-5-yn-2-ylcarbamate (9). ${ }^{1} \mathrm{H}$ NMR (400 MHz, $\mathrm{CDCl}_{3}$ ) $\mathrm{C} 1.41(\mathrm{~s}, 9 \mathrm{H}), 2.76-2.97(\mathrm{~m}, 3 \mathrm{H}), 2.77-2.84(\mathrm{~m}, 1 \mathrm{H}), 3.30(\mathrm{~s}, 1 \mathrm{H}), 4.23-4.31(\mathrm{~m}, 1 \mathrm{H})$, $4.89(\mathrm{~d}, J=8 \mathrm{~Hz}, 1 \mathrm{H}), 7.18-7.33(\mathrm{~m}, 5 \mathrm{H}) ;{ }^{13} \mathrm{C} \mathrm{NMR}\left(100 \mathrm{MHz}, \mathrm{CDCl}_{3}\right) \square 28.7,40.6$, 48.8, 49.0, 79.8, 79.9, 81.7, 127.1, 129.0, 129.8, 137.9, 155.5, 185.9; IR (neat) 3352, $3265,2095,1685 \mathrm{~cm}^{-1}$; HRMS (FAB+) $\mathrm{m} / z$ calc'd for $[\mathrm{M}+\mathrm{H}]^{+} \mathrm{C}_{17} \mathrm{H}_{22} \mathrm{NO}_{3}: 288.1600$,

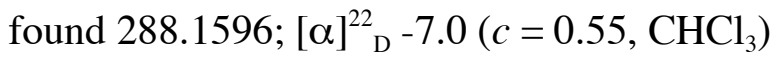

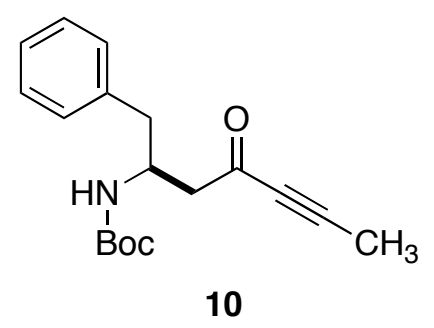

(S)-tert-Butyl 4-Oxo-1-phenylhept-5-yn-2-ylcarbamate (10). ${ }^{1} \mathrm{H}$ NMR $(400 \mathrm{MHz}$, $\left.\mathrm{CDCl}_{3}\right) \square 1.38$ (s, 9H), 2.00 (s, 3H), 2.67 (d, $\left.J=6 \mathrm{~Hz}, 2 \mathrm{H}\right), 2.75-2.92(\mathrm{~m}, 2 \mathrm{H}), 4.23$ (bs, $1 \mathrm{H}), 4.97(\mathrm{~d}, J=8 \mathrm{~Hz}, 1 \mathrm{H}), 7.08-7.28(\mathrm{~m}, 5 \mathrm{H}) ;{ }^{13} \mathrm{C} \mathrm{NMR}\left(100 \mathrm{MHz}, \mathrm{CDCl}_{3}\right) \square 4.5,28.7$, 40.7, 48.7, 49.1, 79.6, 80.6, 91.5, 127.0, 128.9, 129.8, 138.2, 155.5, 186.6; IR (neat) 
3350, 2975, 2929, 2219, 1699, $1164 \mathrm{~cm}^{-1} ;$ HRMS $(\mathrm{FAB}+) \mathrm{m} / \mathrm{z}$ calc'd for $[\mathrm{M}+\mathrm{H}]^{+}$ $\mathrm{C}_{18} \mathrm{H}_{24} \mathrm{NO}_{3}: 302.1756$, found 302.1761; [प] $]_{\mathrm{D}}^{22}-2.0\left(c=0.65, \mathrm{CHCl}_{3}\right)$.

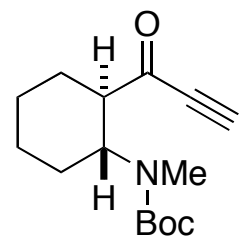

$+/-11$

tert-Butyl Methyl((trans)-2-propioloylcyclohexyl)carbamate (11). ${ }^{1} \mathrm{H}$ NMR (400 $\left.\mathrm{MHz}, \mathrm{CDCl}_{3}, 50{ }^{\circ} \mathrm{C}\right) \square 1.15-1.29(\mathrm{~m}, 2 \mathrm{H}), 1.32-1.73(\mathrm{~m}, 2 \mathrm{H}), 1.46(\mathrm{~s}, 9 \mathrm{H}), 1.79-1.89(\mathrm{~m}$, 3H), 1.93-1.98 (m, 1H), 2.75 (bs, 3H), 2.77-2.88 (m, 1H), 3.25 (s, 1H), 4.00-4.23 (bm, $1 \mathrm{H}) ;{ }^{13} \mathrm{C}$ NMR (125 MHz, $\mathrm{CDCl}_{3}, 50{ }^{\circ} \mathrm{C}$, rotomeric) $\square 24.5,25.1,28.3,28.8,29.7,55.3$, 55.9, 56.8, 79.5, 80.2, 80.8, 155.1, 188.8; IR (neat) 3211, 2933, 2860, 2089, 1690, 1151 $\mathrm{cm}^{-1}$; HRMS (ES+) $m / z$ calc'd for $[\mathrm{M}+\mathrm{H}]^{+} \mathrm{C}_{15} \mathrm{H}_{24} \mathrm{NO}_{3}: 266.1756$, found 266.1757 .

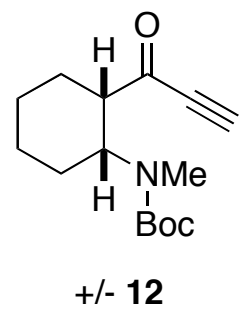

tert-Butyl Methyl((cis)-2-propioloylcyclohexyl)carbamate (12). ${ }^{1} \mathrm{H}$ NMR (400 MHz, $\left.\mathrm{CDCl}_{3}, 50{ }^{\circ} \mathrm{C}\right) \square 1.15-1.8(\mathrm{~m}, 5 \mathrm{H}), 1.46(\mathrm{~s}, 9 \mathrm{H}), 1.87-1.90(\mathrm{~m}, 1 \mathrm{H}), 2.04-2.09(\mathrm{~m}, 1 \mathrm{H})$, 2.14-2.18 (m, 1H), 2.79 (s, 3H), 3.18 (s, 1H), 3.44 (bs, 1H), 3.98 (bs, $1 \mathrm{H}) ;{ }^{13} \mathrm{C}$ NMR (125 $\mathrm{MHz}, \mathrm{CDCl}_{3}, 50{ }^{\circ} \mathrm{C}$, rotomeric) $\square 21.3,25.7,26.2,27.7,28.2,29.5,52.2,56.1,77.3,79.5$, 
82.2, 155.6, 188.6; IR (neat) 3400, 2929, 2858, 2087, 1690, $1148 \mathrm{~cm}^{-1}$; HRMS (ES+) $\mathrm{m} / \mathrm{z}$ calc'd for $[\mathrm{M}+\mathrm{H}]^{+} \mathrm{C}_{15} \mathrm{H}_{24} \mathrm{NO}_{3}: 266.1756$, found 266.1749 .

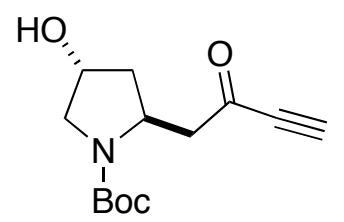

13

(2S,4R)-tert-Butyl 4-Hydroxy-2-(2-oxobut-3-ynyl)pyrrolidine-1-carboxylate (13). ${ }^{1} \mathrm{H}$ NMR (400 MHz, $\left.\mathrm{CDCl}_{3}, 50{ }^{\circ} \mathrm{C}\right) \square 1.42$ (s, 9H), 1.76-1.82 (bm, 1H), 2.12-2.17 (bm, 1H), $2.66(\mathrm{dd}, J=16 \mathrm{~Hz}, 9 \mathrm{~Hz}, 1 \mathrm{H}), 2.90(\mathrm{bs}, 1 \mathrm{H}), 3.20(\mathrm{bs}, 1 \mathrm{H}), 3.31(\mathrm{~s}, 1 \mathrm{H}), 3.33-3.42(\mathrm{~m}$, 2H), 4.27-4.34 (m, 2H); ${ }^{13} \mathrm{C}$ NMR (100 MHz, $\left.\mathrm{CDCl}_{3}\right) \square 28.8,40.7,50.5$, 52.6, 55.0, 69.4, 79.6, 80.4, 82.0, 155.0, 185.4; IR (neat) 3400, 3250, 2990, 2900, 2100, 1700, $1400 \mathrm{~cm}^{-1}$; HRMS (ES+) $m / z$ calc'd for $[\mathrm{M}+\mathrm{H}]^{+} \mathrm{C}_{13} \mathrm{H}_{20} \mathrm{NO}_{4}: 254.1392$, found $254.1389 ;[\square]^{22}{ }_{\mathrm{D}}-55(c$ $\left.=0.89, \mathrm{CHCl}_{3}\right)$.

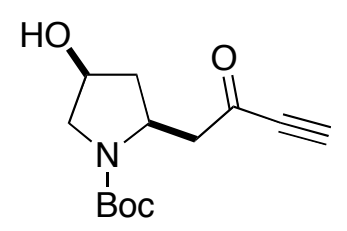

14

(2S,4S)-tert-Butyl 4-Hydroxy-2-(2-oxobut-3-ynyl)pyrrolidine-1-carboxylate (14). ${ }^{1} \mathrm{H}$ NMR (400 MHz, $\left.\mathrm{CDCl}_{3}, 50{ }^{\circ} \mathrm{C}\right) \square 1.47$ (s, 9H), 1.85 (bd, $\left.J=14 \mathrm{~Hz}, 1 \mathrm{H}\right), 2.03(\mathrm{~s}, 1 \mathrm{H})$, $2.26(\mathrm{ddd}, J=14 \mathrm{~Hz}, 9 \mathrm{~Hz}, 5 \mathrm{~Hz}, 1 \mathrm{H}), 3.08(\mathrm{dd}, J=16 \mathrm{~Hz}, 9 \mathrm{~Hz}, 1 \mathrm{H}), 3.23(\mathrm{~s}, 1 \mathrm{H}), 3.38$ (bd, $J=12 \mathrm{~Hz}, 2 \mathrm{H}), 3.59$ (dd, $J=12 \mathrm{~Hz}, 5 \mathrm{~Hz}, 1 \mathrm{H}), 4.27-4.34(\mathrm{~m}, 1 \mathrm{H}), 4.43-4.47$ (m, $1 \mathrm{H}) ;{ }^{13} \mathrm{C}$ NMR $\left(100 \mathrm{MHz}, \mathrm{CDCl}_{3}\right) \square 28.9,39.7,51.1,53.1,55.4,70.8,78.8,80.4,82.2$, 
154.6, 186.0; IR (neat) 3400, 2975, 2090, 1680, $1400 \mathrm{~cm}^{-1}$; HRMS (ES+) $m / z$ calc'd for $[\mathrm{M}+\mathrm{H}]^{+} \mathrm{C}_{13} \mathrm{H}_{20} \mathrm{NO}_{4}: 254.1392$, found 254.1393; [D] ${ }_{\mathrm{D}}^{22}-4\left(c=0.6, \mathrm{CHCl}_{3}\right)$.

\section{Enaminone characterization data (15-25):}

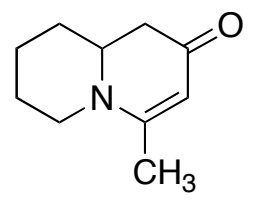

15

4-Methyl-7,8,9,9a-tetrahydro-1 $\boldsymbol{H}$-quinolizin-2(6H)-one (15). ${ }^{1} \mathrm{H}$ NMR (400 $\mathrm{MHz}$, $\left.\mathrm{CDCl}_{3}\right) \square 1.37-1.86(\mathrm{~m}, 6 \mathrm{H}), 1.95$ (s, 3H), $2.25(\mathrm{dd}, J=10.6 \mathrm{~Hz}, 16.5 \mathrm{~Hz}, 1 \mathrm{H}), 2.48(\mathrm{dd}, J$ $=16.5 \mathrm{~Hz}, 5.7 \mathrm{~Hz}, 1 \mathrm{H}), 2.76-2.82(\mathrm{~m}, 1 \mathrm{H}), 3.28-3.38(\mathrm{~m}, 1 \mathrm{H}), 3.74-3.78(\mathrm{~m}, 1 \mathrm{H}), 4.95(\mathrm{~s}$, $1 \mathrm{H}) ;{ }^{13} \mathrm{C}$ NMR (100 MHz, $\left.\mathrm{CDCl}_{3}\right) \square 21.6,24.1,26.1,31.8,43.2,48.5,59.0,102.1,163.4$, 191.8; IR (neat) 3444, 2934, 2855, 1626, $1557 \mathrm{~cm}^{-1}$; HRMS (FAB+) $\mathrm{m} / z$ calc'd for $[\mathrm{M}+\mathrm{H}]^{+} \mathrm{C}_{10} \mathrm{H}_{16} \mathrm{NO}: 166.1232$, found 166.1234 .

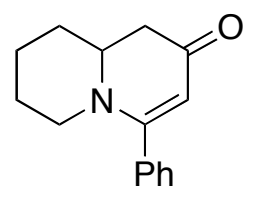

16

4-Phenyl-7,8,9,9a-tetrahydro-1H-quinolizin-2(6H)-one (16). ${ }^{1} \mathrm{H}$ NMR (500 $\mathrm{MHz}$, $\left.\mathrm{CDCl}_{3}\right) \square 1.46-1.50(\mathrm{~m}, 2 \mathrm{H}), 1.56-1.60(\mathrm{~m}, 1 \mathrm{H}), 1.75-1.80(\mathrm{~m}, 2 \mathrm{H}), 1.88-1.98(\mathrm{~m}, 1 \mathrm{H})$, $2.47(\mathrm{dd}, J=16 \mathrm{~Hz}, 11 \mathrm{~Hz}, 1 \mathrm{H}), 2.60-2.66(\mathrm{~m}, 2 \mathrm{H}), 3.50-3.58(\mathrm{~m}, 2 \mathrm{H}), 5.09(\mathrm{~s}, 1 \mathrm{H})$, 7.28-7.46 (m, 5H); ${ }^{13} \mathrm{C}$ NMR (100 MHz, $\left.\mathrm{CDCl}_{3}\right) \square 23.8,25.9,31.3,42.6,50.3,58.6$, 
103.3, 127.0, 128.5, 128.9, 136.7, 165.9, 191.5; IR (neat) 2932, 2853, 1640, $1541 \mathrm{~cm}^{-1}$; HRMS (FAB+) $m / z$ calc'd for $[\mathrm{M}+\mathrm{H}]^{+} \mathrm{C}_{15} \mathrm{H}_{18} \mathrm{NO}: 228.1388$, found 228.1400 .

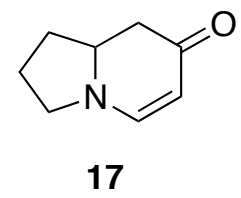

2,3,8,8a-Tetrahydroindolizin-7(1H)-one (17). ${ }^{1} \mathrm{H}$ NMR (400 MHz, $\left.\mathrm{CDCl}_{3}\right) \square 1.61-1.70$ (m, 1H), 1.86-1.95 (m, 1H), 2.05-2.10 (m, 1H), 2.21-2.44 (m, 3H), 3.42-3.54 (m, 2H), 3.66-3.76 (m, 1H), $4.91(\mathrm{~d}, J=7 \mathrm{~Hz}, 1 \mathrm{H}), 7.19(\mathrm{~d}, J=7 \mathrm{~Hz}, 1 \mathrm{H}) ;{ }^{13} \mathrm{C} \mathrm{NMR}(100 \mathrm{MHz}$, $\left.\mathrm{CDCl}_{3}\right) \square 24.8,33.2,41.9,49.7,58.5,97.5,150.4,192.5$; IR (neat) 3423, 297, 2879, $1617,1559 \mathrm{~cm}^{-1}$; HRMS (FAB+) $\mathrm{m} / z$ calc'd for $[\mathrm{M}+\mathrm{H}]^{+} \mathrm{C}_{8} \mathrm{H}_{12} \mathrm{NO}: 138.1901$, found 138.0911.

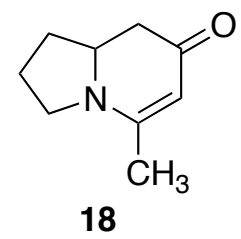

5-Methyl-2,3,8,8a-tetrahydroindolizin-7(1H)-one (18). ${ }^{1} \mathrm{H}$ NMR (400 $\left.\mathrm{MHz}, \mathrm{CDCl}_{3}\right)$ 1.61-1.70 (m, 1H), 1.82-1.95 (m, 1H), $2.01(\mathrm{~s}, 3 \mathrm{H}), 2.05-2.10(\mathrm{~m}, 1 \mathrm{H}), 2.21-2.42(\mathrm{~m}$, $3 \mathrm{H}), 3.42-3.47(\mathrm{~m}, 1 \mathrm{H}), 3.52-3.57(\mathrm{~m}, 1 \mathrm{H}), 3.70-3.80(\mathrm{~m}, 1 \mathrm{H}), 4.92(\mathrm{~s}, 1 \mathrm{H}) ;{ }^{13} \mathrm{C} \mathrm{NMR}$ (100 MHz, $\left.\mathrm{CDCl}_{3}\right) \square 20.9,24.0,33.1,41.6,47.3,59.3,98.4,161.2,191.7$; IR (neat) 3397, $2918,1597,1531 \mathrm{~cm}^{-1}$; HRMS (ES+) $\mathrm{m} / z$ calc'd for $[\mathrm{M}+\mathrm{H}]^{+} \mathrm{C}_{9} \mathrm{H}_{14} \mathrm{NO}: 152.1075$, found 152.1093. 


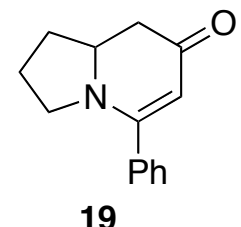

5-Phenyl-2,3,8,8a-tetrahydroindolizin-7(1H)-one (19). ${ }^{1} \mathrm{H} \mathrm{NMR}\left(400 \mathrm{MHz}, \mathrm{CDCl}_{3}\right)$ $1.75-1.85(\mathrm{~m}, 1 \mathrm{H}), 1.85-2.00(\mathrm{~m}, 1 \mathrm{H}), 2.01-2.09(\mathrm{~m}, 1 \mathrm{H}), 2.30-2.40(\mathrm{~m}, 1 \mathrm{H}), 2.41-2.54$ (m, 2H), 3.27-3.31 (m, 1H), 3.48-3.52 (m, 1H), 4.04-4.07 (m, 1H), 5.10, (s, 1H), 7.38$7.45(\mathrm{~m}, 5 \mathrm{H}) ;{ }^{13} \mathrm{C} \mathrm{NMR}\left(100 \mathrm{MHz}, \mathrm{CDCl}_{3}\right) \square 24.9,32.4,41.9,49.7,59.3,100.4,128.0$, 128.9, 130.2, 136.6, 163.3, 192.4; IR (neat) 3427, 2964, 2877, 1611, 1518, $1471 \mathrm{~cm}^{-1}$; HRMS (ES+) $m / z$ calc'd for $[\mathrm{M}+\mathrm{H}]^{+} \mathrm{C}_{14} \mathrm{H}_{16} \mathrm{NO}: 214.1232$, found 214.1227.

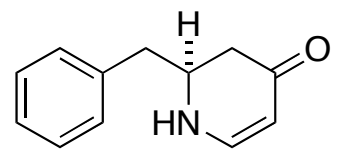

20

(S)-2-Benzyl-2,3-dihydropyridin-4(1H)-one (20). Spectral data was identical to the reported values: $[\square]_{D}^{22}-150,\left(c=0.65, \mathrm{CHCl}_{3}\right)$; literature value $=:[\square]_{\mathrm{D}}^{22}-151,(c=$ $\left.0.225, \mathrm{CHCl}_{3}\right)^{2}$

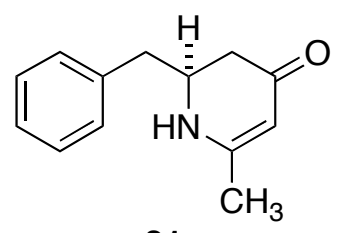

21

(S)-2-Benzyl-6-methyl-2,3-dihydropyridin-4(1H)-one (21). $\quad{ }^{1} \mathrm{H} \quad \mathrm{NMR}(400 \mathrm{MHz}$, $\left.\mathrm{CDCl}_{3}\right) \square 1.92(\mathrm{~s}, 3 \mathrm{H}), 2.36(\mathrm{dd}, J=16 \mathrm{~Hz}, 11.5 \mathrm{~Hz}, 1 \mathrm{H}), 2.48(\mathrm{dd}, J=16 \mathrm{~Hz}, 5 \mathrm{~Hz}, 1 \mathrm{H})$, 2.84-2.93 (m, 2H), 3.81-3.85 (m, 1H), $4.82(\mathrm{~s}, 1 \mathrm{H}), 4.98(\mathrm{~s}, 1 \mathrm{H}), 7.19-7.38(\mathrm{~m}, 5 \mathrm{H}) ;{ }^{13} \mathrm{C}$ 
NMR (100 MHz, $\left.\mathrm{CDCl}_{3}\right) \square 21.6,40.8,41.7,54.7,100.1,127.5,129.4,129.6,137.3$, 161.2, 191.6; IR (neat) 3244, 1608, 1597, $1531 \mathrm{~cm}^{-1}$; HRMS (FAB+) $\mathrm{m} / z$ calc'd for $[\mathrm{M}+\mathrm{H}]^{+} \mathrm{C}_{13} \mathrm{H}_{16} \mathrm{NO}: 202.1232$, found 202.1234; [D] ${ }_{\mathrm{D}}^{22}-190\left(c=1.6, \mathrm{CHCl}_{3}\right)$.

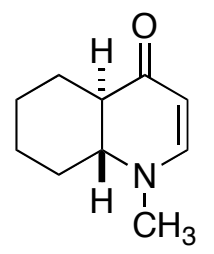

22

(trans)-1-Methyl-4a,5,6,7,8,8a-hexahydroquinolin-4(1H)-one (22). ${ }^{1} \mathrm{H} \quad \mathrm{NMR} \quad(500$ $\left.\mathrm{MHz}, \mathrm{CDCl}_{3}\right) \square 0.95-1.04(\mathrm{~m}, 1 \mathrm{H}), 1.10-1.28(\mathrm{~m}, 2 \mathrm{H}), 1.30-1.39(\mathrm{~m}, 1 \mathrm{H}), 1.70-1.85(\mathrm{~m}$, 2H), 2.00-2.08 (m, 1H), 2.13-2.15 (m, 1H), 2.32-2.35 (m, 1H), 2.89 (s, 3H), 2.92-3.06 $(\mathrm{m}, 1 \mathrm{H}) 4.96(\mathrm{~d}, J=7 \mathrm{~Hz}, 1 \mathrm{H}), 6.98(\mathrm{~d}, J=7 \mathrm{~Hz}, 1 \mathrm{H}) ;{ }^{13} \mathrm{C} \mathrm{NMR}\left(100 \mathrm{MHz}, \mathrm{CDCl}_{3}\right) \square$ 23.5, 23.8, 24.0, 29.2, 38.5, 47.4, 61.1, 97.3, 154.2, 193.0; IR (neat) 3417, 2924, 2856, $1634,1590,1191 \mathrm{~cm}^{-1}$; HRMS (ES+) $m / z$ calc'd for $[\mathrm{M}+\mathrm{H}]^{+} \mathrm{C}_{10} \mathrm{H}_{16} \mathrm{NO}: 166.1232$, found 166.1235.

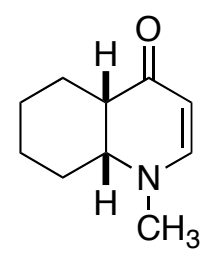

23

(cis)-1-Methyl-4a,5,6,7,8,8a-hexahydroquinolin-4(1H)-one (23). ${ }^{1} \mathrm{H}$ NMR $(500 \mathrm{MHz}$, $\left.\mathrm{CDCl}_{3}\right) \square 1.23-1.32(\mathrm{~m}, 2 \mathrm{H}), 1.37-1.42(\mathrm{~m}, 2 \mathrm{H}), 1.50-1.57(\mathrm{~m}, 1 \mathrm{H}), 1.57-1.66(\mathrm{~m}, 1 \mathrm{H})$, 1.77-1.87 (m, 1H), 2.10-2.18 (m, 1H), 2.56-2.64 (m, 1H), 2.99 (s, 3H), 3.30-3.38 (m, $1 \mathrm{H}), 4.87(\mathrm{~d}, J=7 \mathrm{~Hz}, 1 \mathrm{H}), 6.83(\mathrm{~d}, J=7 \mathrm{~Hz}, 1 \mathrm{H}) ;{ }^{13} \mathrm{C} \mathrm{NMR}\left(100 \mathrm{MHz}, \mathrm{CDCl}_{3}\right) \square 22.8$, 
23.8, 24.3, 29.0, 30.6, 45.0, 60.1, 96.7, 153.6, 192.0; IR (neat) 2930, 2855, 1631, 1590, $1207 \mathrm{~cm}^{-1}$; HRMS (ES+) $\mathrm{m} / z$ calc'd for $[\mathrm{M}+\mathrm{H}]^{+} \mathrm{C}_{10} \mathrm{H}_{16} \mathrm{NO}: 166.1232$, found 166.1227 .

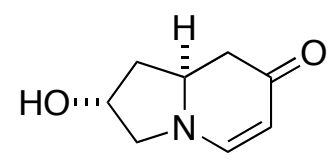

24

(2R,8aS)-2-Hydroxy-2,3,8,8a-tetrahydroindolizin-7(1H)-one (24). ${ }^{1} \mathrm{H}$ NMR (400 $\left.\mathrm{MHz}, \mathrm{CDCl}_{3}\right) \square 1.84(\mathrm{ddd}, J=15.5 \mathrm{~Hz}, 11.3 \mathrm{~Hz}, 4.2 \mathrm{~Hz}, 1 \mathrm{H}), 2.03(\mathrm{dd}, J=12.9 \mathrm{~Hz}, 5.6$ $\mathrm{Hz}, 1 \mathrm{H}), 2.36(\mathrm{dd}, J=16.3 \mathrm{~Hz}, 16.3 \mathrm{~Hz}), 1 \mathrm{H}), 2.47(\mathrm{dd}, J=16.1 \mathrm{~Hz}, 5.1 \mathrm{~Hz}, 1 \mathrm{H}), 3.00$ (bs, 1H), $3.54(\mathrm{~d}, J=11.9 \mathrm{~Hz}, 1 \mathrm{H}), 3.75(\mathrm{dd}, J=11.9 \mathrm{~Hz}, 4.4 \mathrm{~Hz}, 1 \mathrm{H}), 4.10-4.19(\mathrm{~m}$, $1 \mathrm{H}), 4.65(\mathrm{t}, J=4.1 \mathrm{~Hz}, 1 \mathrm{H}), 5.00(\mathrm{~d}, J=7 \mathrm{~Hz}, 1 \mathrm{H}), 7.24(\mathrm{~d}, J=7 \mathrm{~Hz}, 1 \mathrm{H}) ;{ }^{13} \mathrm{C}$ NMR $\left(100 \mathrm{MHz}, \mathrm{CDCl}_{3}\right) \square 41.3,42.1,56.4,58.6,70.3,97.8,151.2,192.7$; IR (neat) 3400,1580

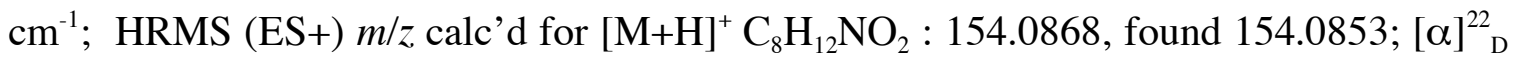
$-490\left(c=0.45, \mathrm{CHCl}_{3}\right)$.

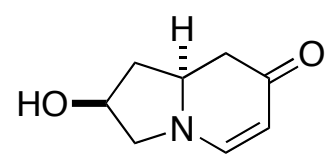

25

(2R,8aR)-2-Hydroxy-2,3,8,8a-tetrahydroindolizin-7(1H)-one (ent-25). ${ }^{1} \mathrm{H}$ NMR (400 $\left.\mathrm{MHz}, \mathrm{D}_{3} \mathrm{CCOCD}_{3}\right) \square 1.74(\mathrm{ddd}, J=15.5 \mathrm{~Hz}, 9 \mathrm{~Hz}, 6.5 \mathrm{~Hz}, 1 \mathrm{H}), 2.05-2.08(\mathrm{~m}, 1 \mathrm{H}), 2.22$ (dd, $J=15.8 \mathrm{~Hz}, 5 \mathrm{~Hz}, 1 \mathrm{H}), 2.38(\mathrm{dd}, J=15.8 \mathrm{~Hz}, 15.8 \mathrm{~Hz}, 1 \mathrm{H}), 2.51(\mathrm{ddd}, J=6.4 \mathrm{~Hz}$, $12.6 \mathrm{~Hz}, 6.4 \mathrm{~Hz}, 1 \mathrm{H}), 3.36(\mathrm{dd}, J=10.7 \mathrm{~Hz}, 5.5 \mathrm{~Hz}, 1 \mathrm{H}), 3.69(\mathrm{dd}, J=10.7 \mathrm{~Hz}, 6.5 \mathrm{~Hz}$, 1H), 3.74-3.81 (m, 1H), 4.50-4.56 (m, 1H), $4.75(\mathrm{~d}, J=7.4 \mathrm{~Hz}, 1 \mathrm{H}), 7.30(\mathrm{~d} J=7.4 \mathrm{~Hz}$; ${ }^{13} \mathrm{C}$ NMR $\left(100 \mathrm{MHz}, \mathrm{D}_{3} \mathrm{CCOCD}_{3}\right) \square 41.4,42.2,57.0,57.2,70.0,97.2,150.1,190.6$; IR 
(neat) $3320,2871,1614,1560 \mathrm{~cm}^{-1}$; HRMS (FAB+) $\mathrm{m} / z$ calc'd for $[\mathrm{M}+\mathrm{H}]^{+} \mathrm{C}_{8} \mathrm{H}_{12} \mathrm{NO}_{2}$ : 154.0868, found 154.0862; [[] ${ }_{\mathrm{D}}^{22}+780\left(c=0.90, \mathrm{CHCl}_{3}\right)$.

Additional Compounds (26, 29, 30):

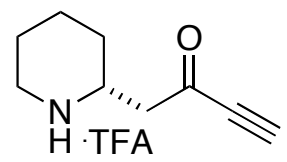

26

(R)-1-(Piperidin-2-yl)but-3-yn-2-one 2,2,2-Trifluoroacetic Acid (26). ${ }^{1} \mathrm{H}$ NMR (400 $\left.\mathrm{MHz}, \mathrm{CHCl}_{3}\right) \square 1.51-1.62(\mathrm{~m}, 1 \mathrm{H}), 1.70(\mathrm{dd}, J=24 \mathrm{~Hz}, 12 \mathrm{~Hz}, 1 \mathrm{H}), 1.77-1.99(\mathrm{~m}, 4 \mathrm{H})$, 3.00 (dd, $J=18 \mathrm{~Hz}, 6 \mathrm{~Hz}, 2 \mathrm{H}), 2.25$ (bd, $J=18 \mathrm{~Hz}, 1 \mathrm{H}), 3.38$ (s, 1H), 3.45-3.57 (bm, 2H), 9.22 (bd, $J=70 \mathrm{~Hz}, 2 \mathrm{H}$-exchangable); ${ }^{13} \mathrm{C}$ NMR (100 MHz, $\mathrm{CDCl}_{3}$ ) $\square 22.5,22.6$, 28.7, 45.5, 48.1, 52.8, 81.0, 81.3, 183.2; IR (neat) 2958, 2097, 1678, 1203, $1136 \mathrm{~cm}^{-1}$; HRMS (ES+) $m / z$ calc'd for $[\mathrm{M}+\mathrm{H}]^{+} \mathrm{C}_{9} \mathrm{H}_{14} \mathrm{NO}$ : 152.1075 , found 152.1060 .

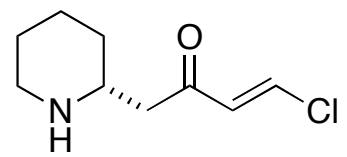

29

( $\boldsymbol{R}, \boldsymbol{E})$-4-Chloro-1-(piperidin-2-yl)but-3-en-2-one (29). ${ }^{1} \mathrm{H} \mathrm{NMR}\left(400 \mathrm{MHz}, \mathrm{CD}_{3} \mathrm{OD}\right)$ $1.55-1.80(\mathrm{~m}, 3 \mathrm{H}), 1.81-1.99(\mathrm{~m}, 3 \mathrm{H}), 2.96-3.17(\mathrm{~m}, 3 \mathrm{H}), 3.30-3.41(\mathrm{~m}, 2 \mathrm{H}), 3.50-3.64$ $(\mathrm{m}, 1 \mathrm{H}), 6.66(\mathrm{~d}, J=13.7 \mathrm{~Hz}, 1 \mathrm{H}), 7.70(\mathrm{~d}, J=13.7 \mathrm{~Hz}, 1 \mathrm{H}) ;{ }^{13} \mathrm{C} \mathrm{NMR}(100 \mathrm{MHz}$, $\left.\mathrm{CD}_{3} \mathrm{OD}\right) \square 22.0,22.3,42.9,45.0,52.9,132.4,139.2,194.8$; IR (neat) 3400, 2951, 1674, $1585 \mathrm{~cm}^{-1}$; HRMS (FAB+) $\mathrm{m} / z$ calc'd for $[\mathrm{M}+\mathrm{H}]^{+} \mathrm{C}_{9} \mathrm{H}_{15} \mathrm{NOCl}: 188.0842$, found 188.0827; [ []$_{\mathrm{D}}^{22}+22\left(c=0.32, \mathrm{CHCl}_{3}\right)$. 


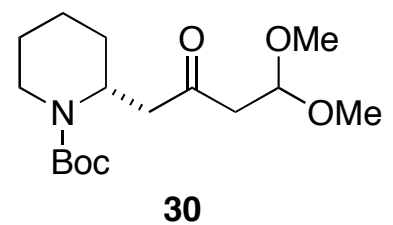

(R)-tert-Butyl 2-(4,4-Dimethoxy-2-oxobutyl)piperidine-1-carboxylate (30). ${ }^{1} \mathrm{H}$ NMR (400 MHz, CDCl $)$ ) $1.38-1.68(\mathrm{~m}, 6 \mathrm{H}), 1.46(\mathrm{~s}, 9 \mathrm{H}), 2.63(\mathrm{dd}, J=15 \mathrm{~Hz}, 6.3 \mathrm{~Hz}, 1 \mathrm{H})$, 2.72-2.79 (m, 4H), $3.36(\mathrm{~s}, 6 \mathrm{H}), 3.99(\mathrm{bs}, 1 \mathrm{H}), 4.73(\mathrm{bs}, 1 \mathrm{H}), 4.77(\mathrm{t}, J=5.6 \mathrm{~Hz}, 1 \mathrm{H}) ;{ }^{13} \mathrm{C}$ NMR $\left(100 \mathrm{MHz}, \mathrm{CDCl}_{3}\right) \square$ 19.3, 25.7, 28.6, 28.8, 44.8, 47.0, 47.3, 54.2, 54.4, 80.0, 102.1, 155.2, 206.2; IR (neat) 3400, 2089, $1643 \mathrm{~cm}^{-1}$; HRMS (ES+) $\mathrm{m} / z$ calc'd for

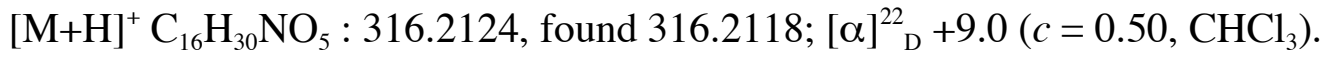

\section{References:}

1. Comins, D. L.; LaMunyon, D. H. J. Org. Chem. 1992, 57, 5807-5809.

2. Comins, D. L.; Zhang, Y.; Joseph, S. P. Org. Lett. 1999, 1, 657-659. 


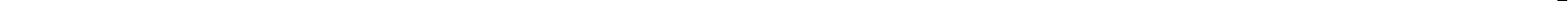



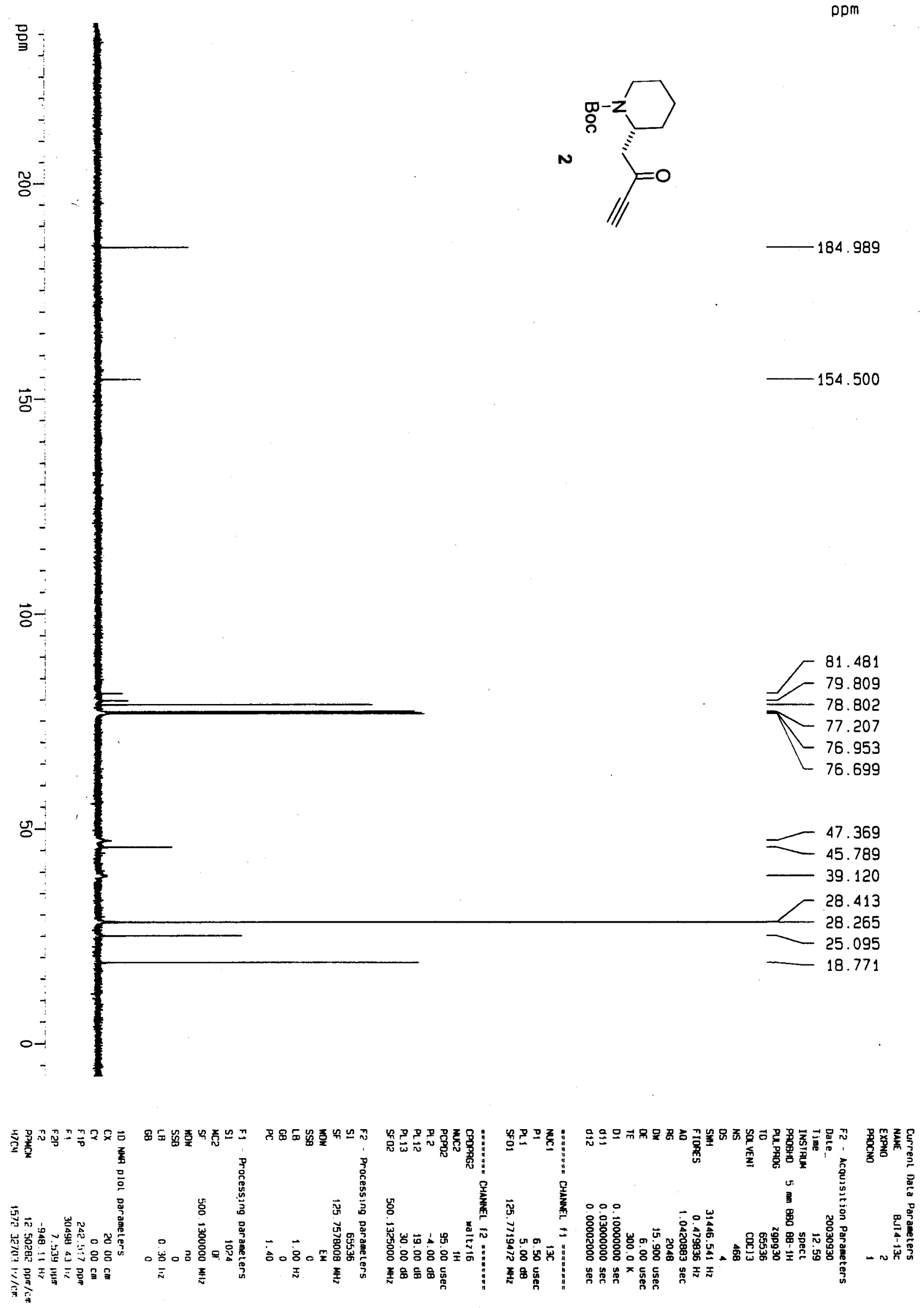


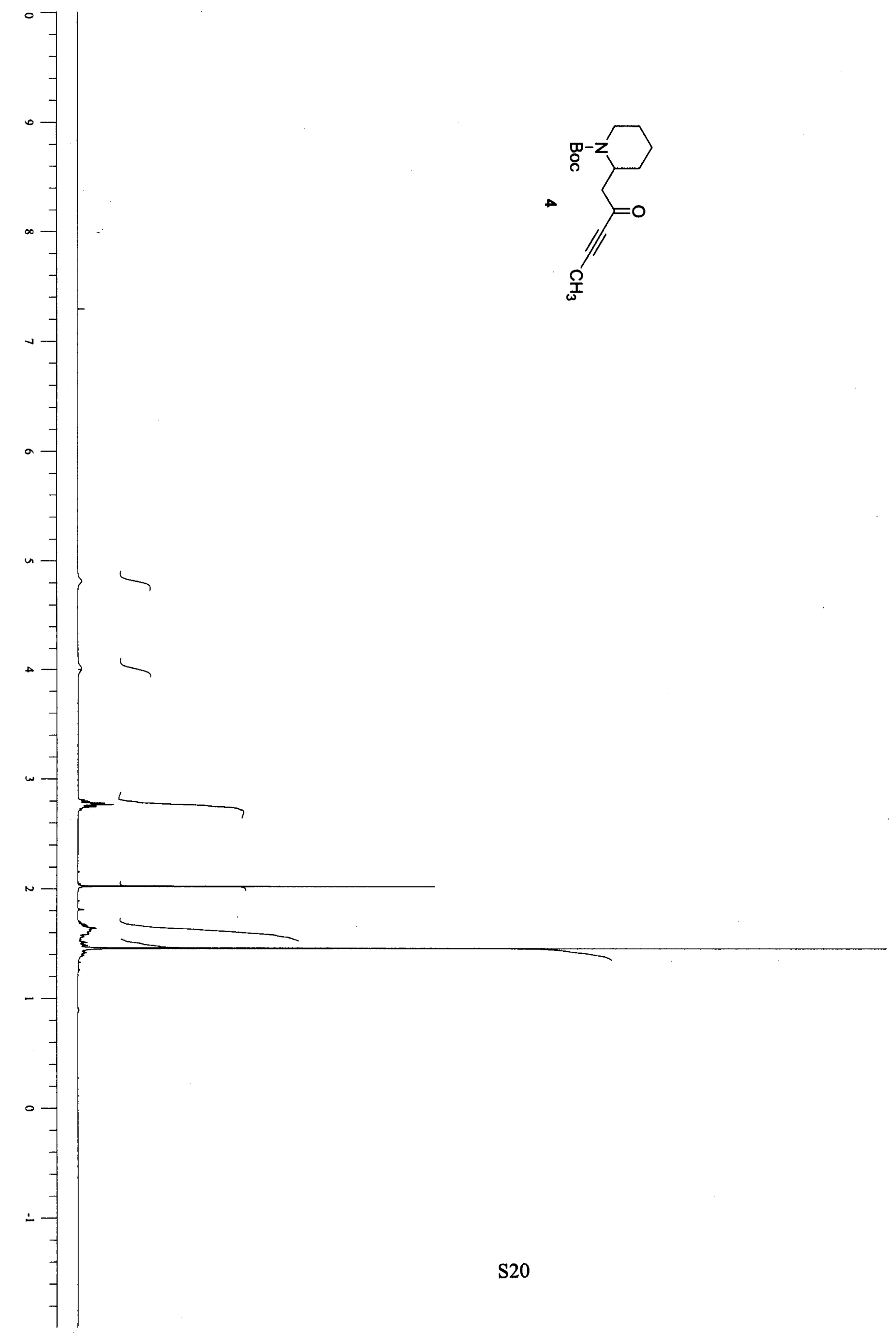




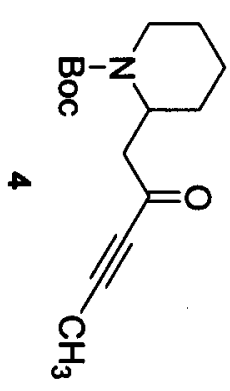

$-185.725$

$-154.545$

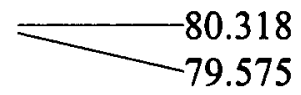

79.575
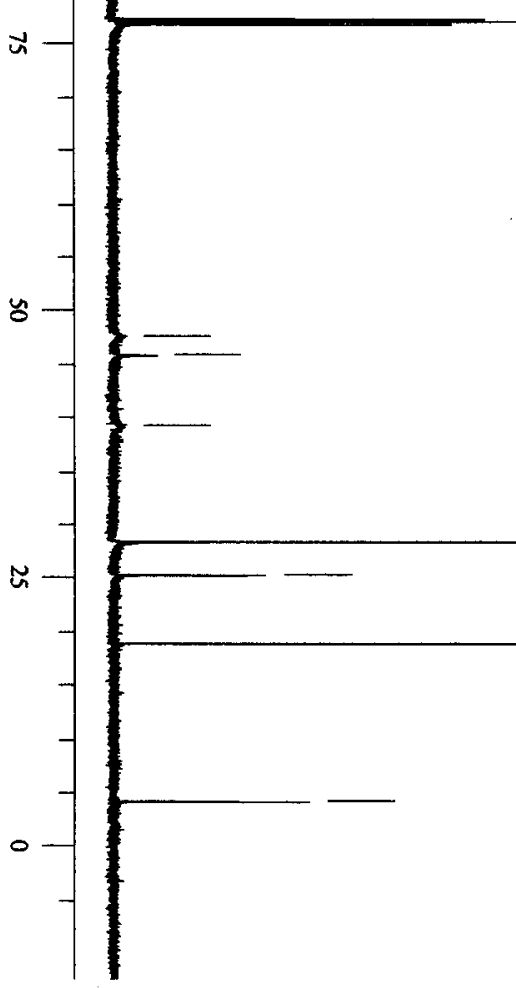

47.503

45.695

39.248

28.253

25.152

18.767 


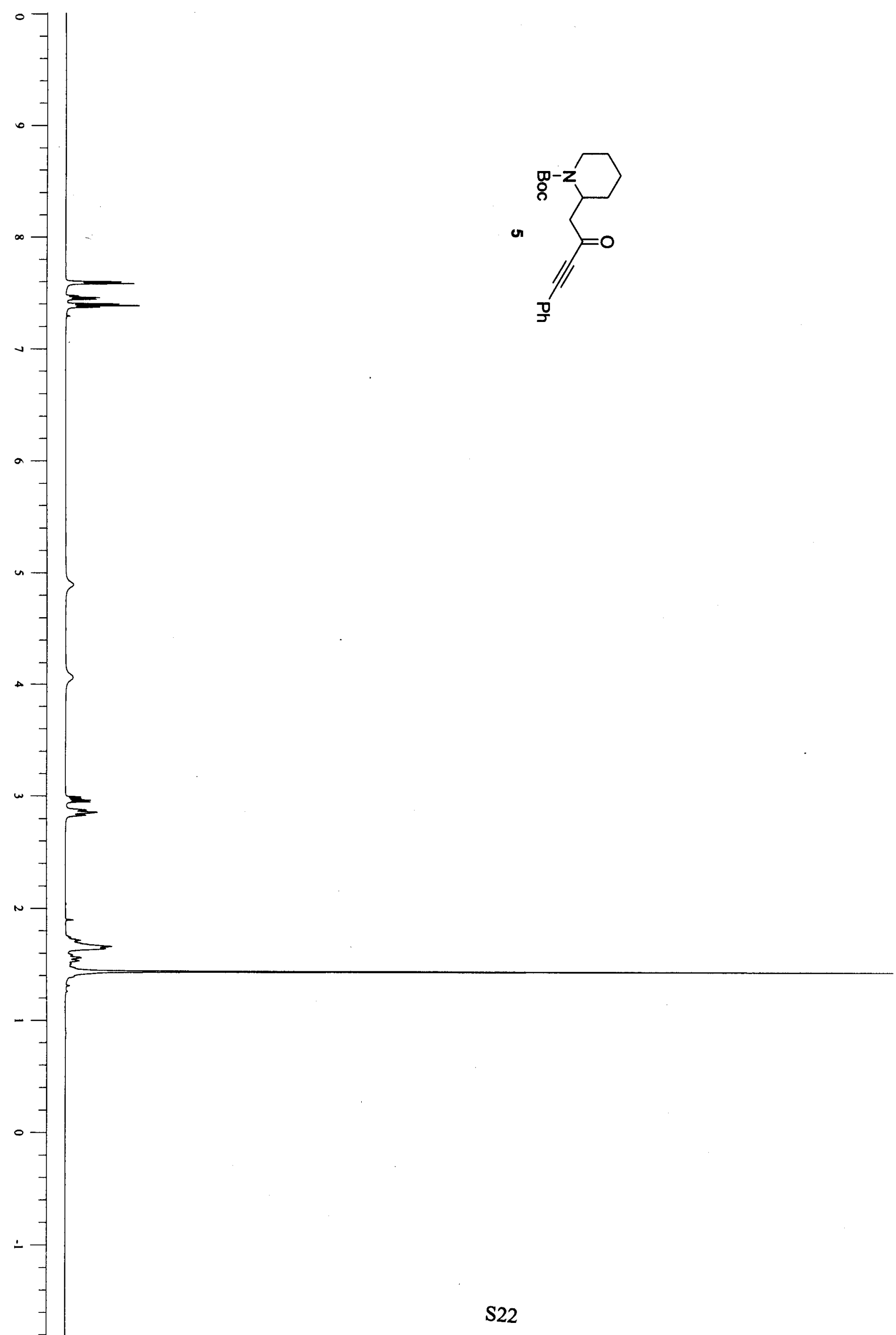




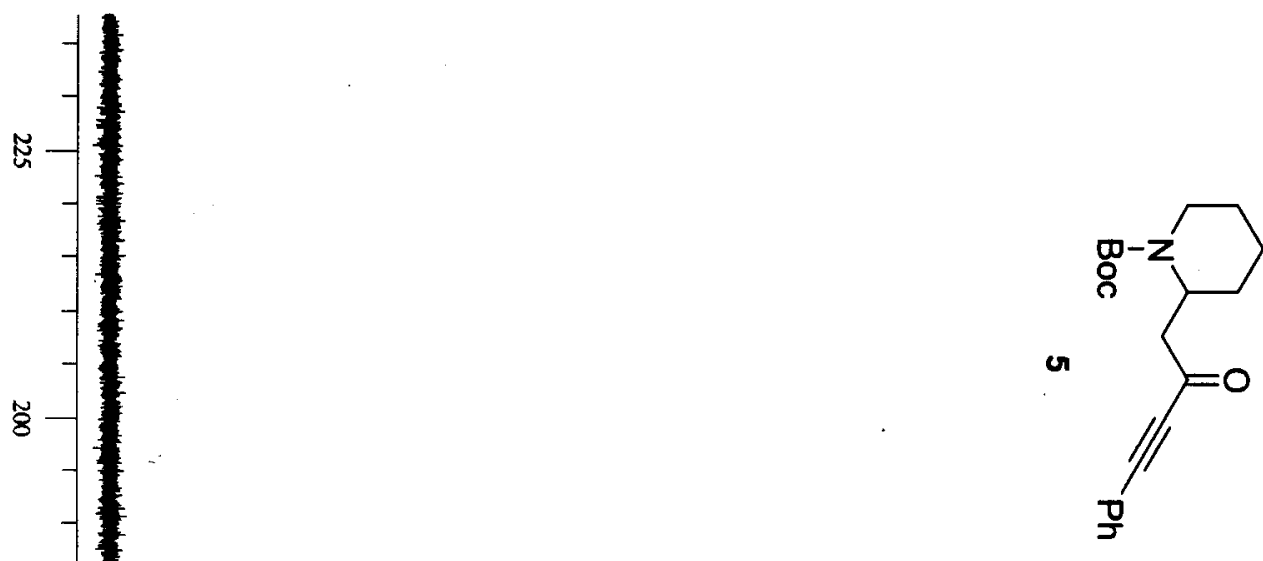

$-185.618$

ज

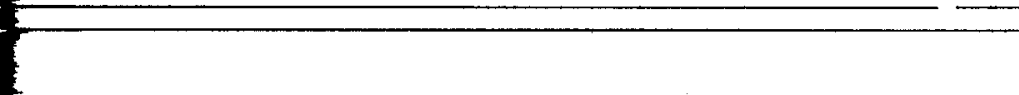

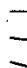

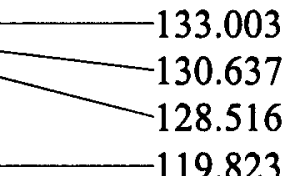

154.537 119.823

$-91.024$ 88.011 $-79.668$

ज

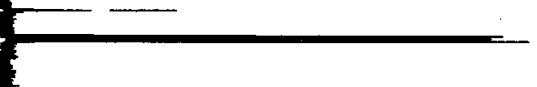

$\longrightarrow$
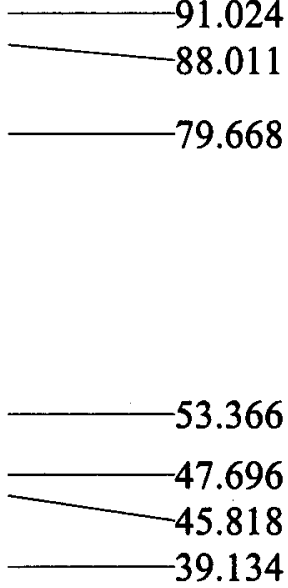

28.25

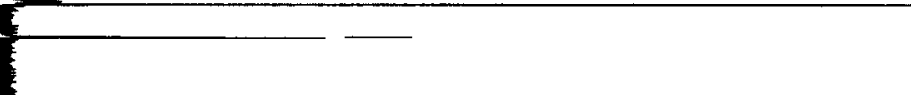

25.183

18.850 


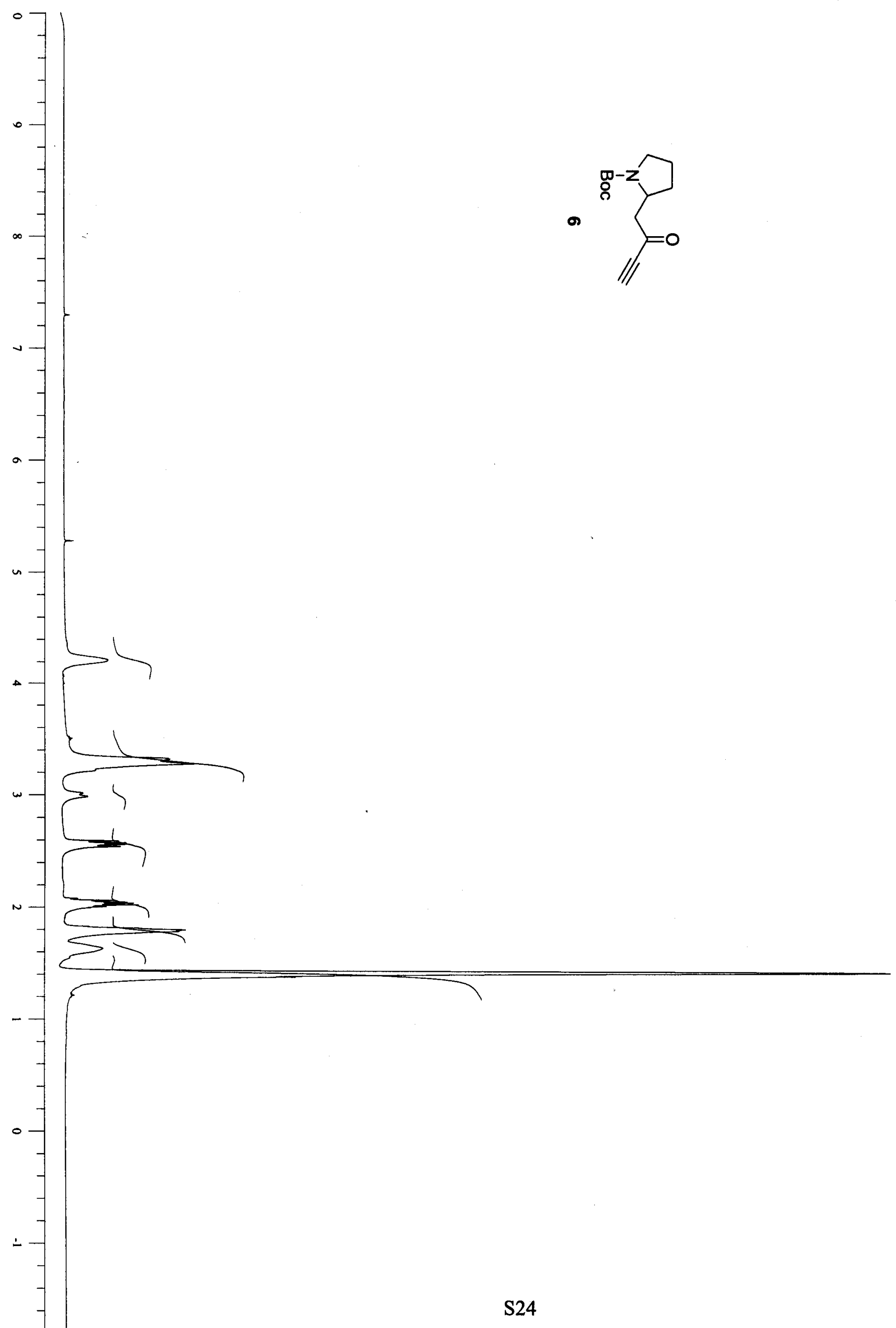



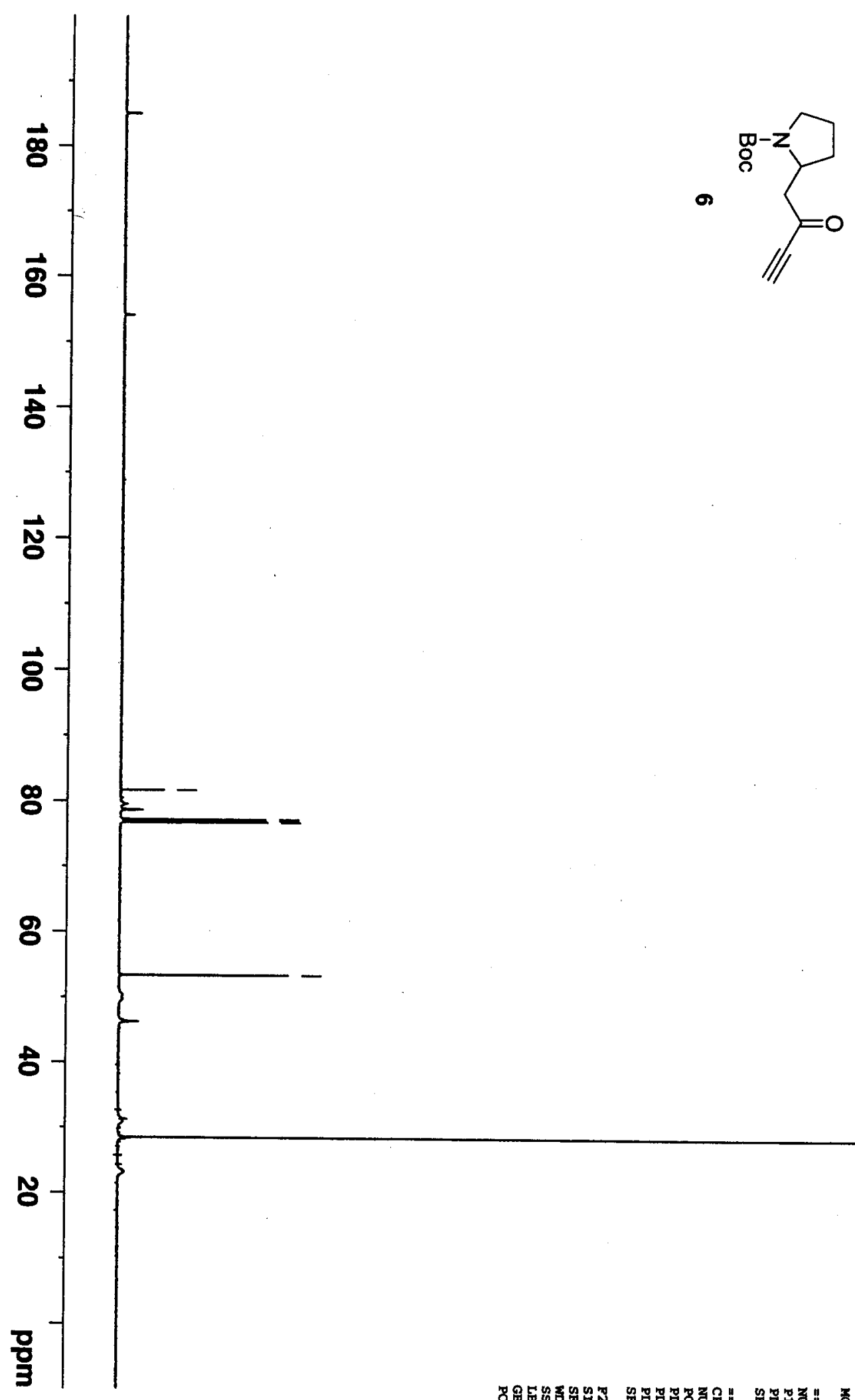


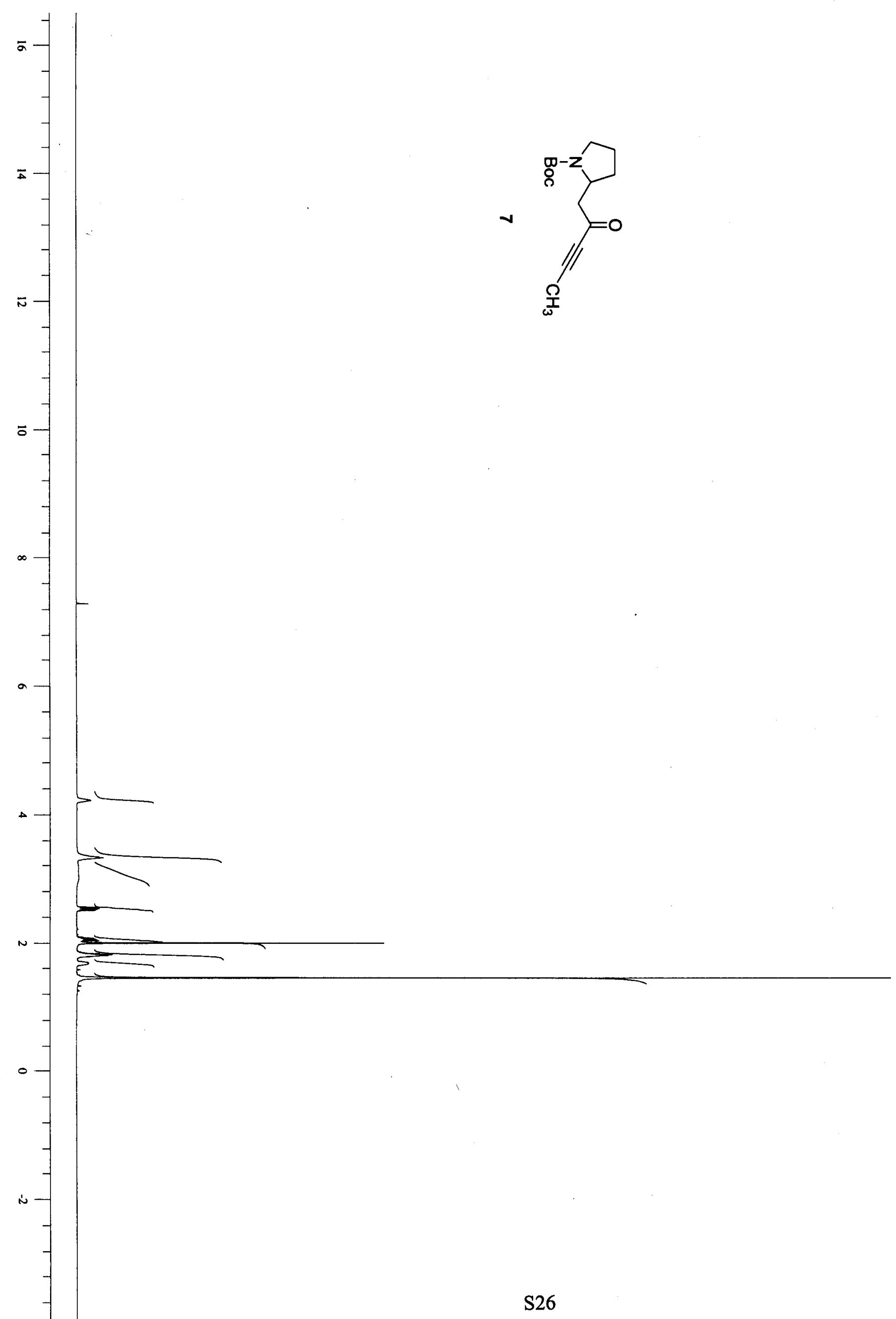




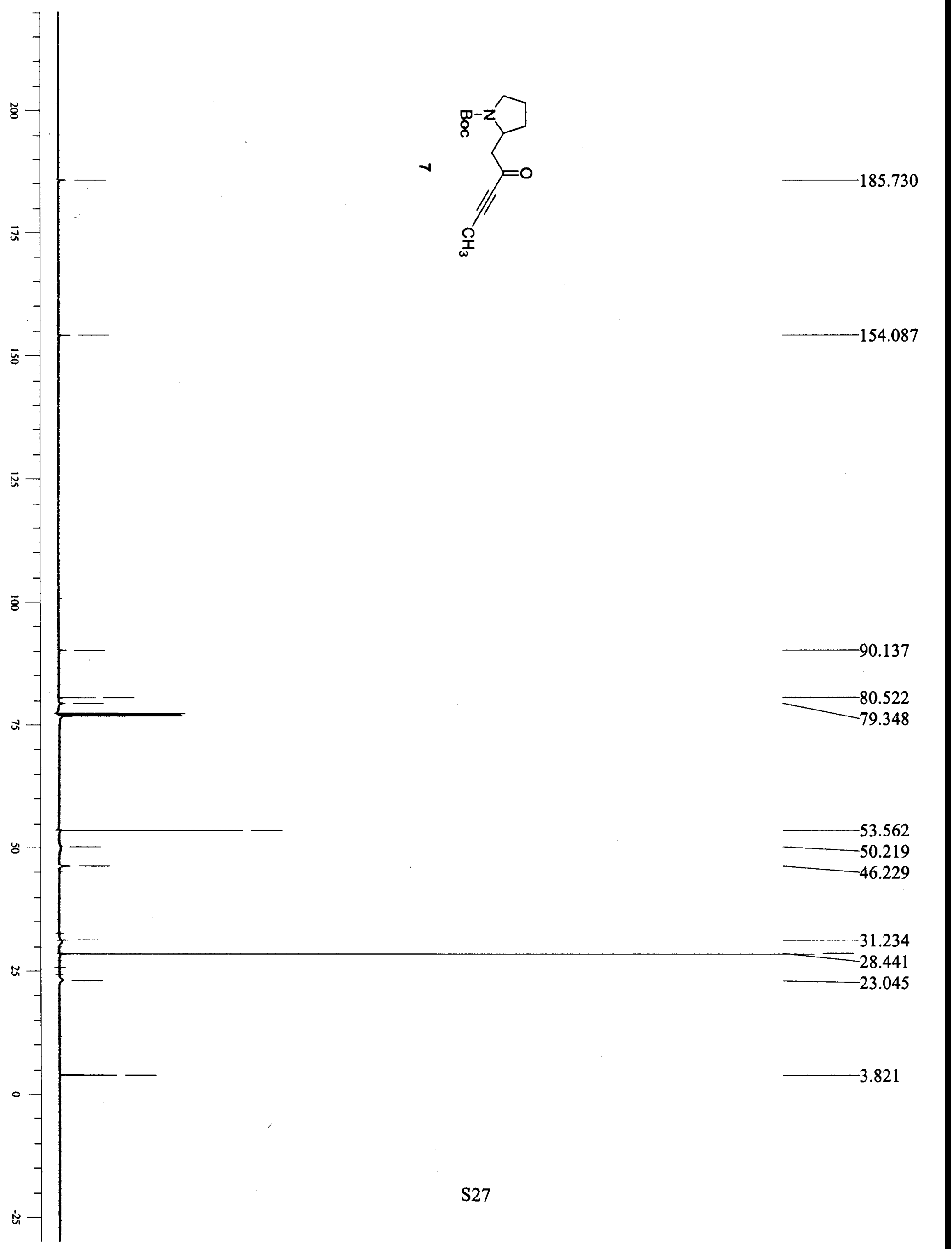




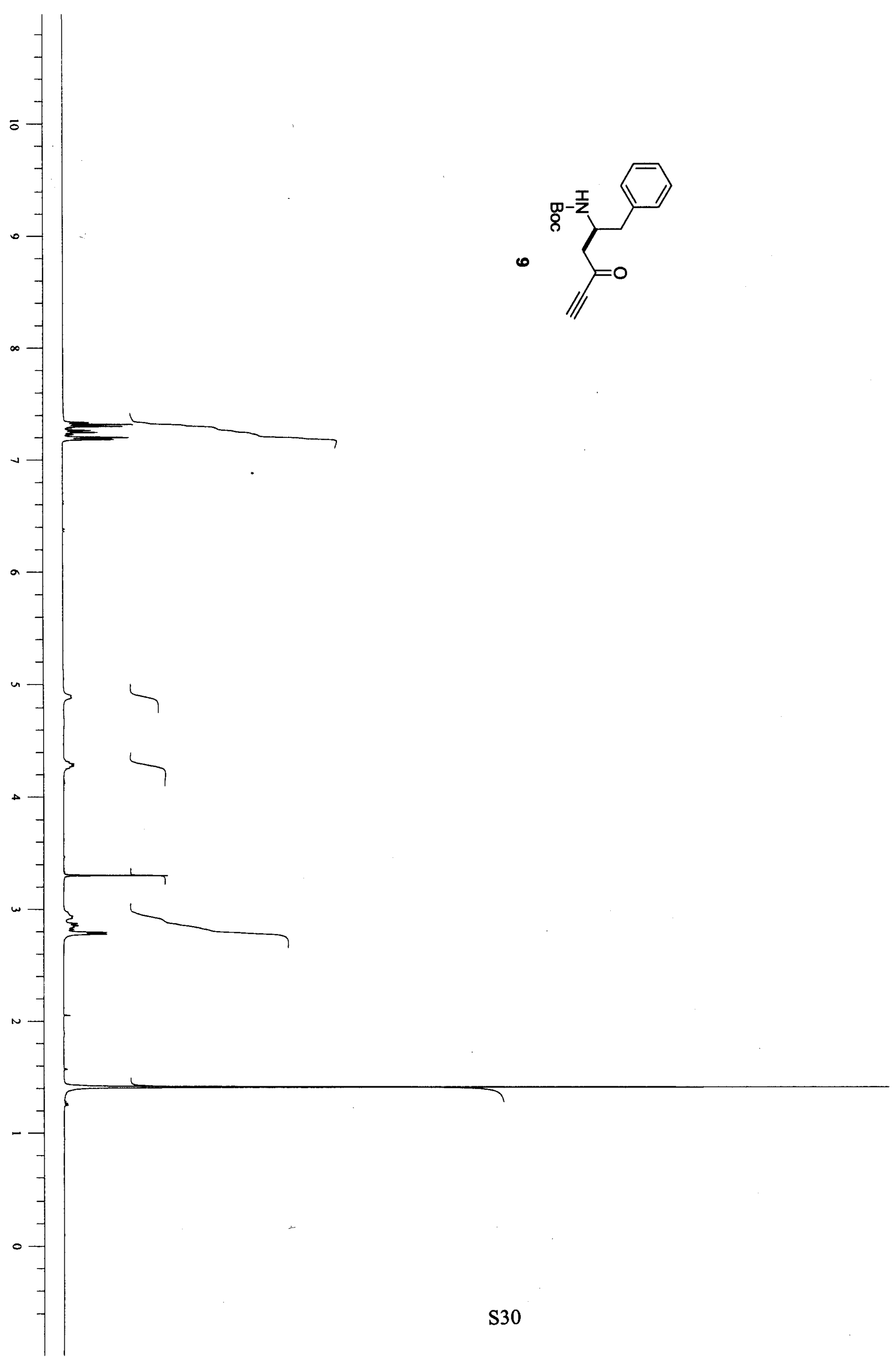




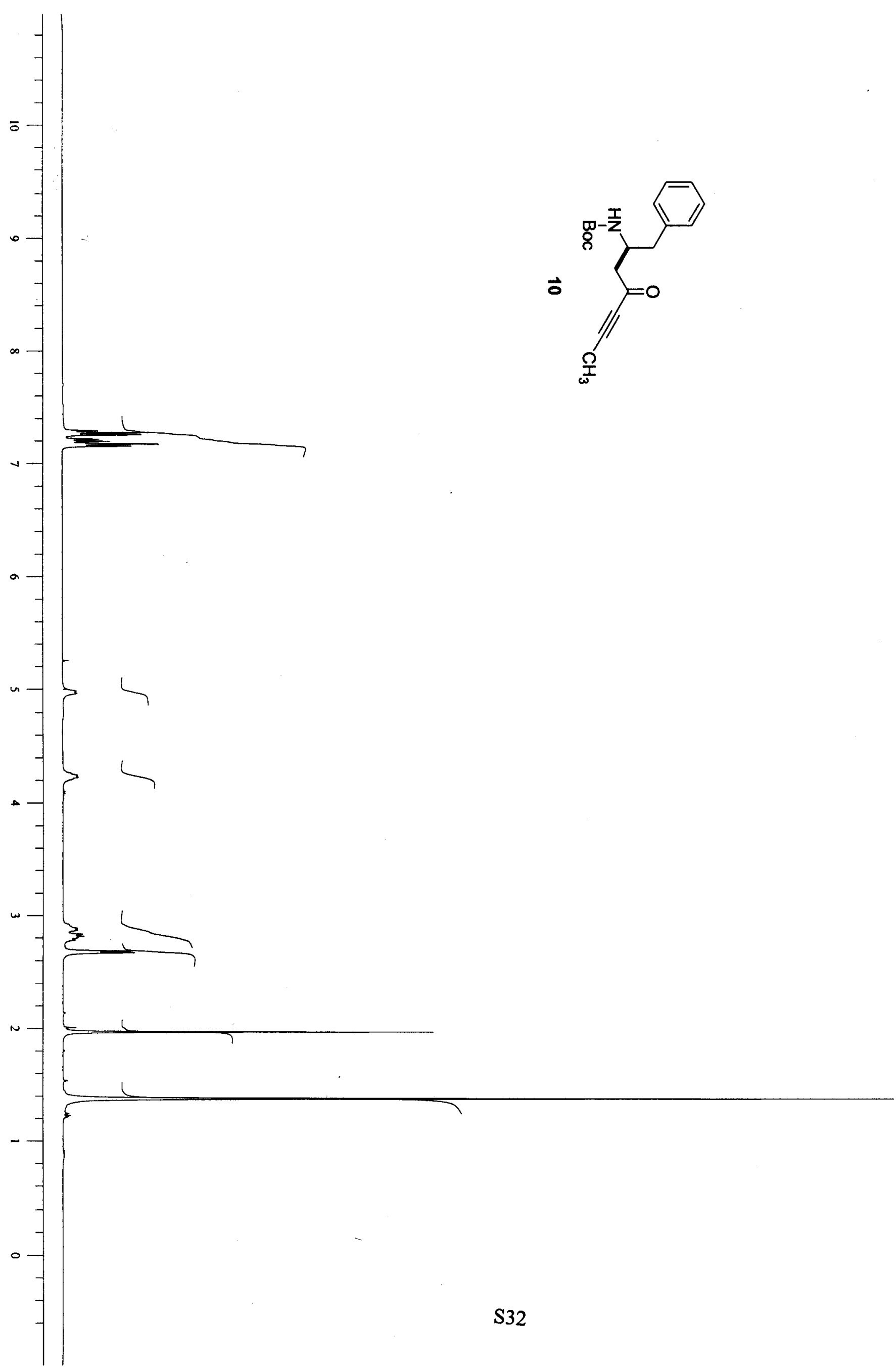



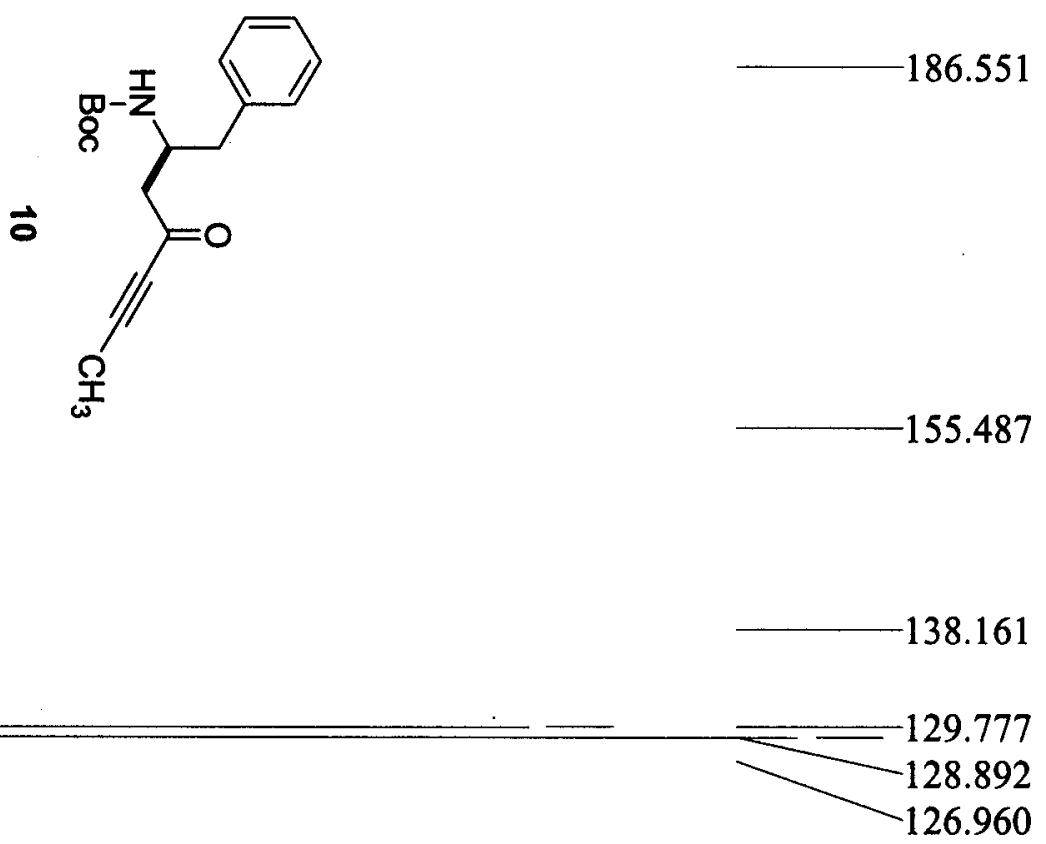

91.532
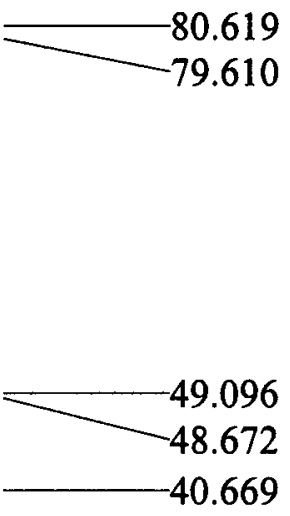

$-28.719$

$\tilde{n}$

$$
\text { . }
$$




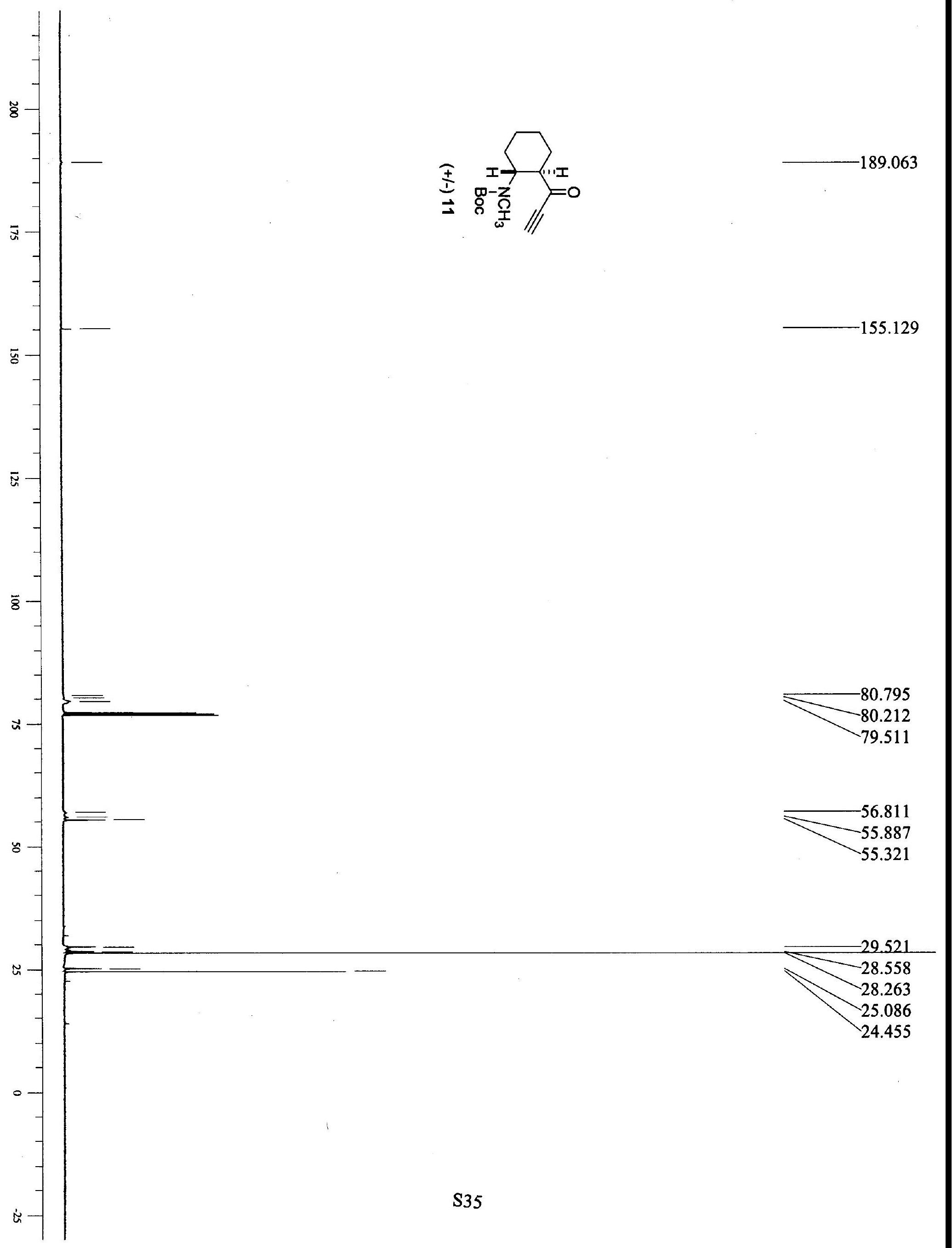



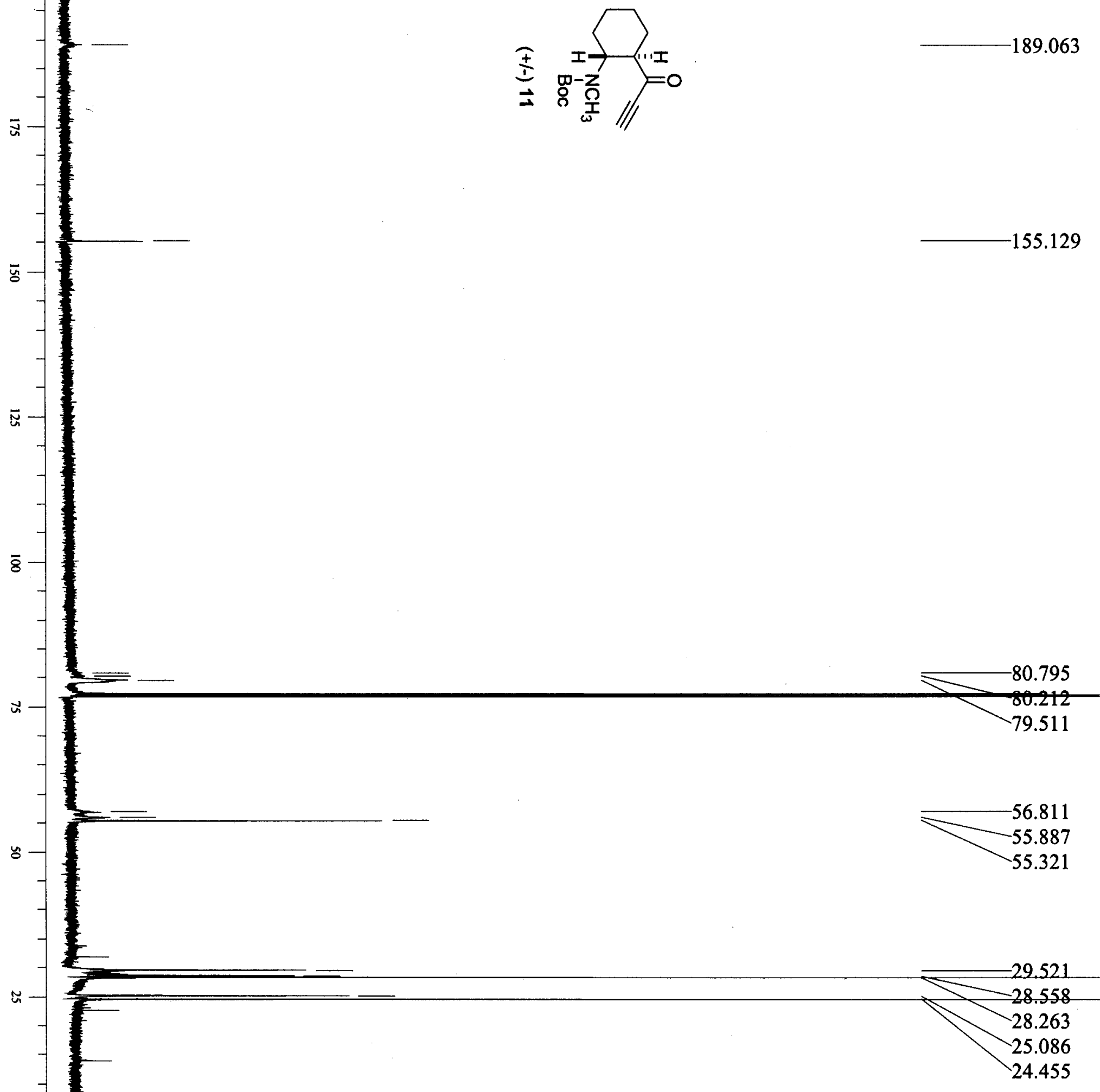

$\circ-$ 


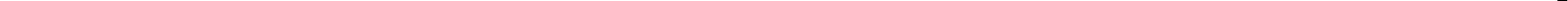



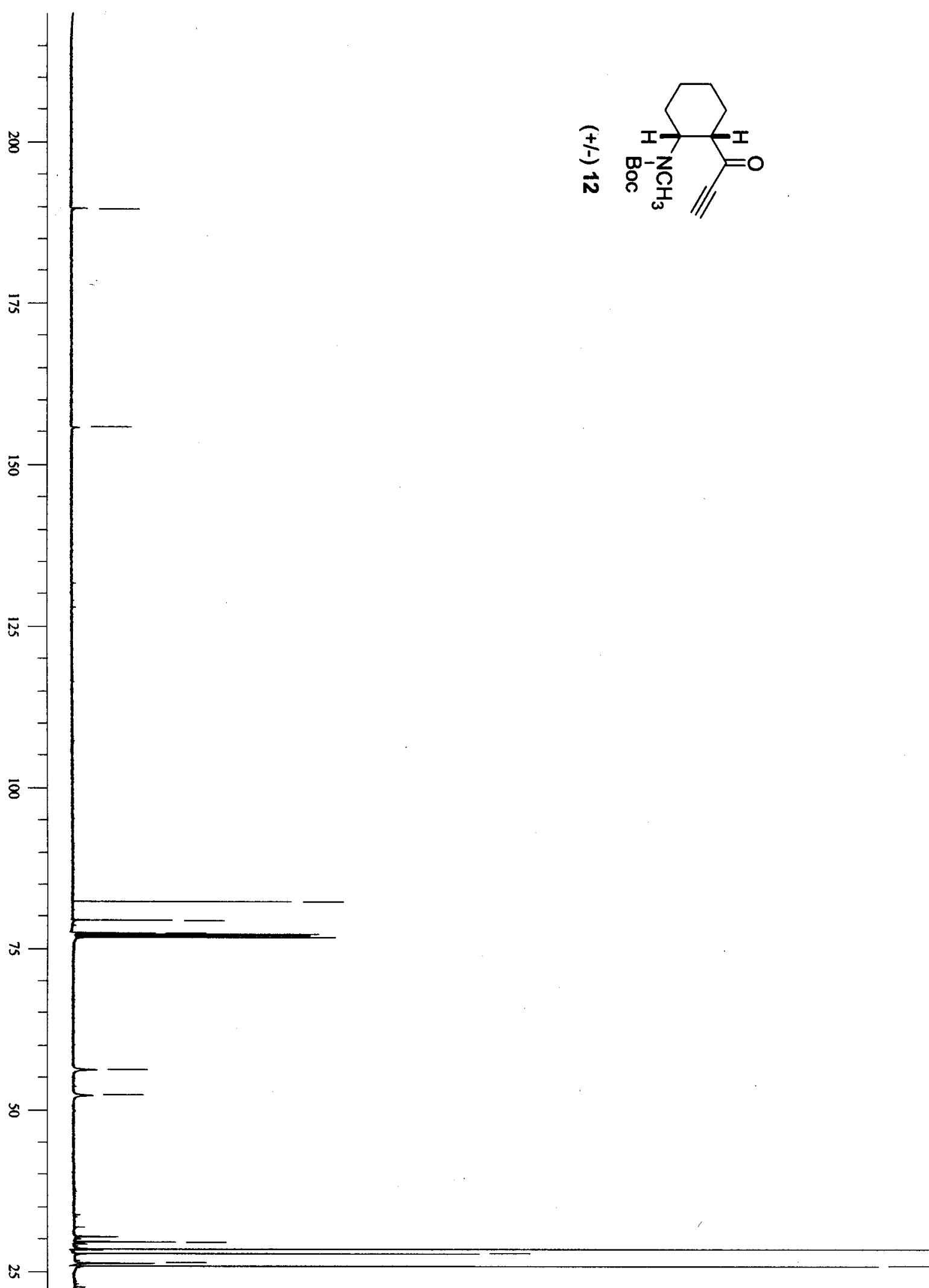

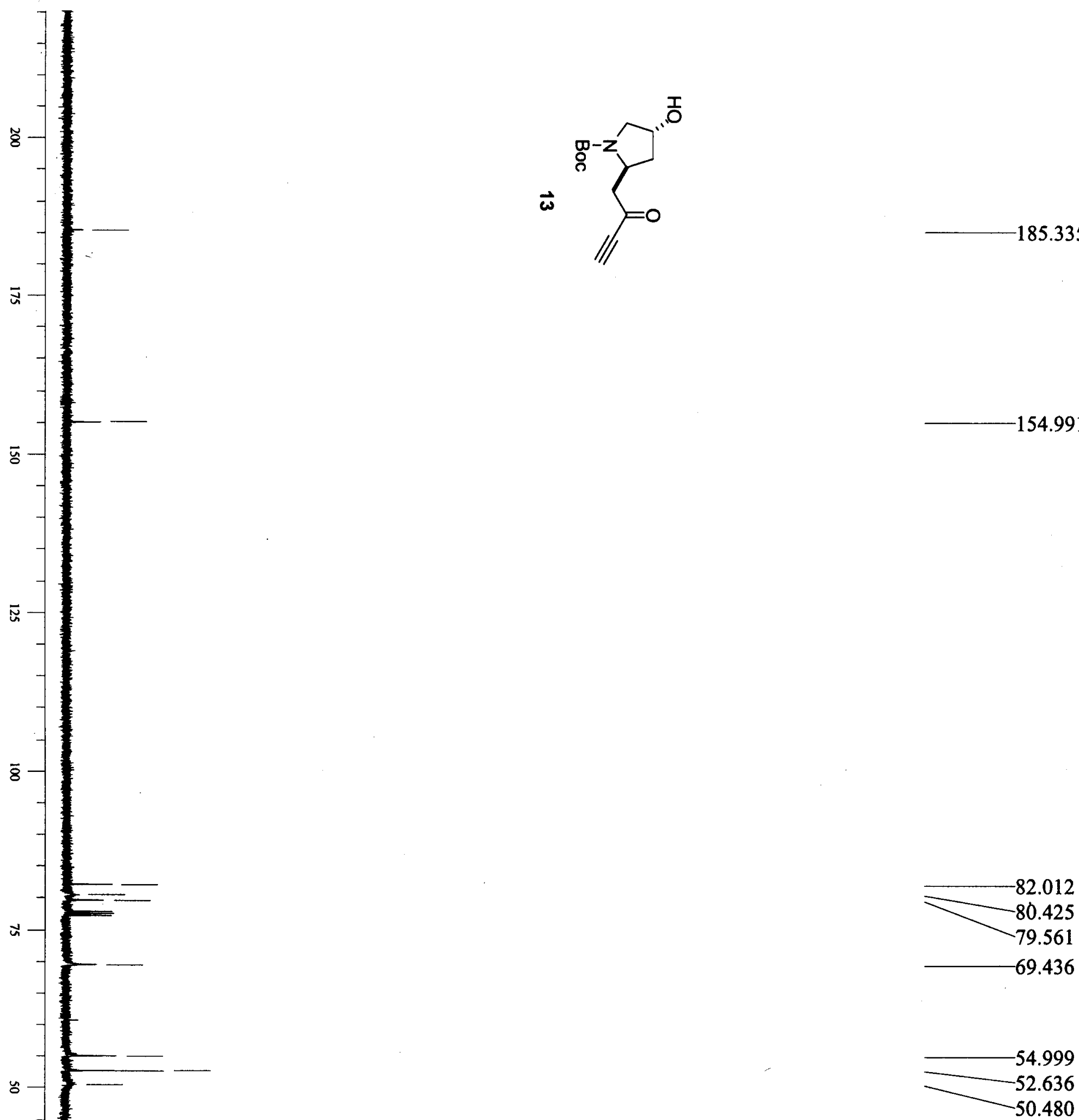

$-40.669$

$-28.791$ 


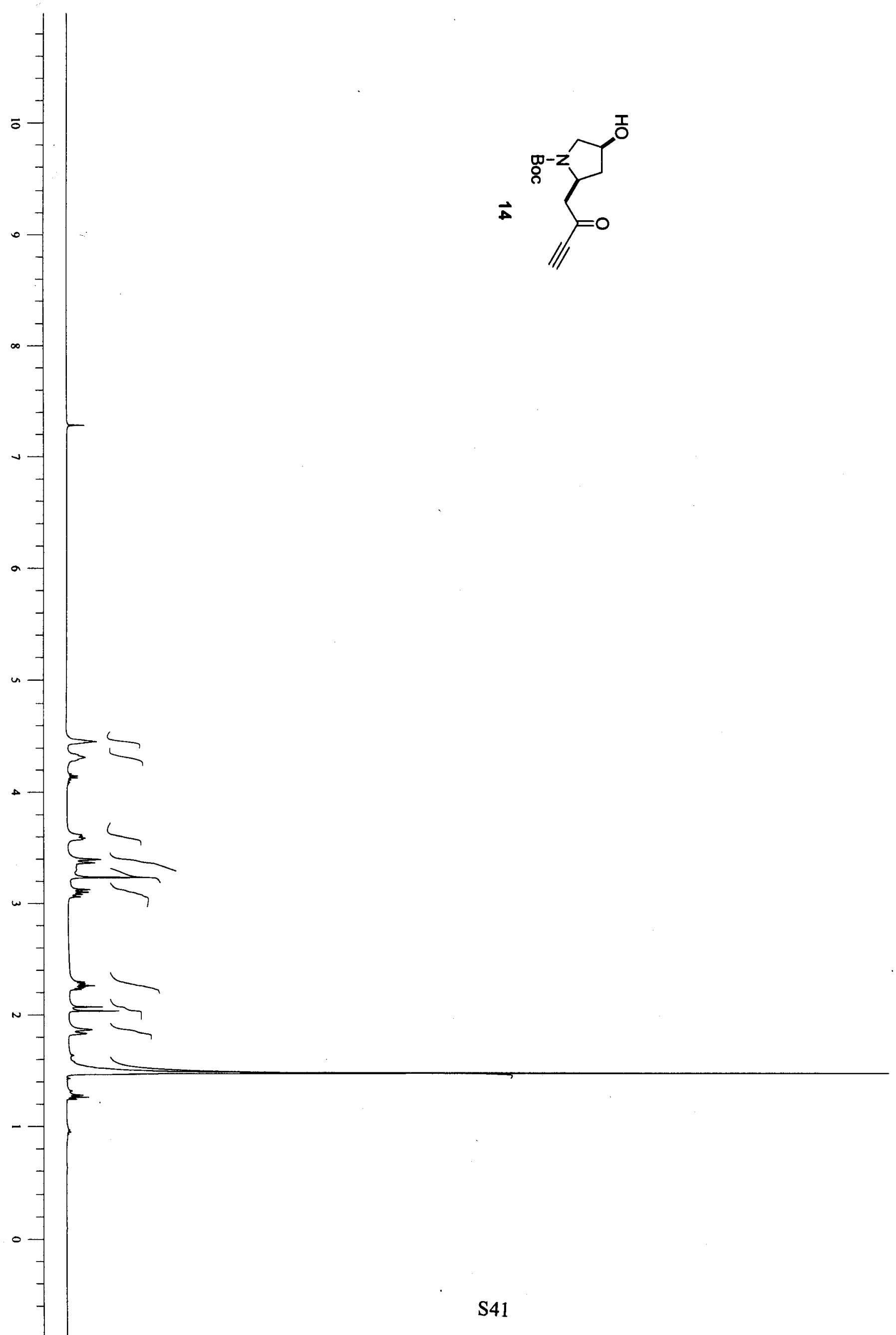



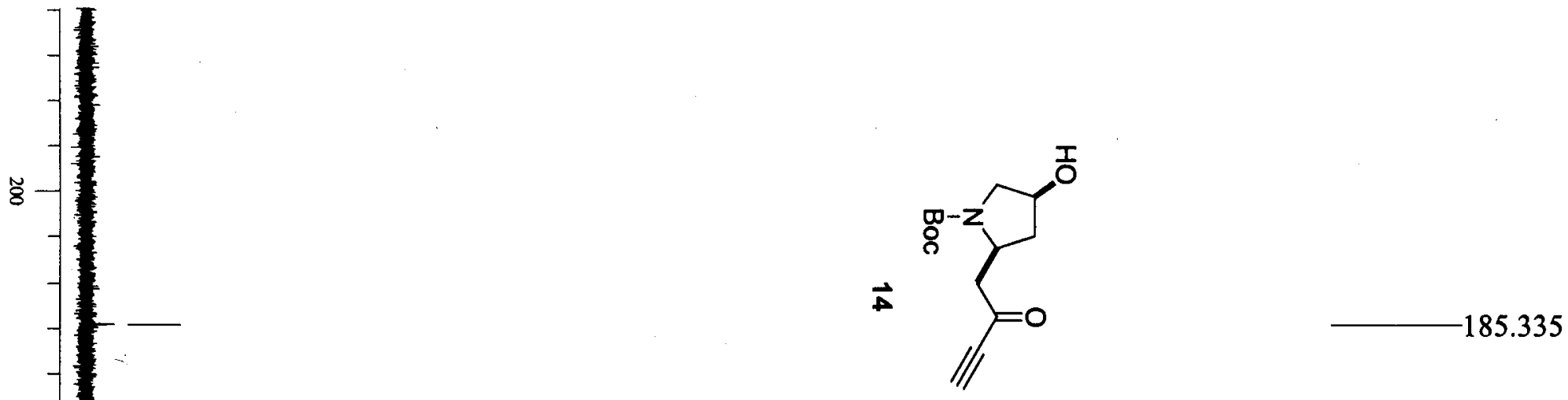

$\overline{4}$

ธิ

154.991

$\overrightarrow{8}$

$\bar{\sim}$

$\overline{8}$

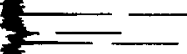

जั
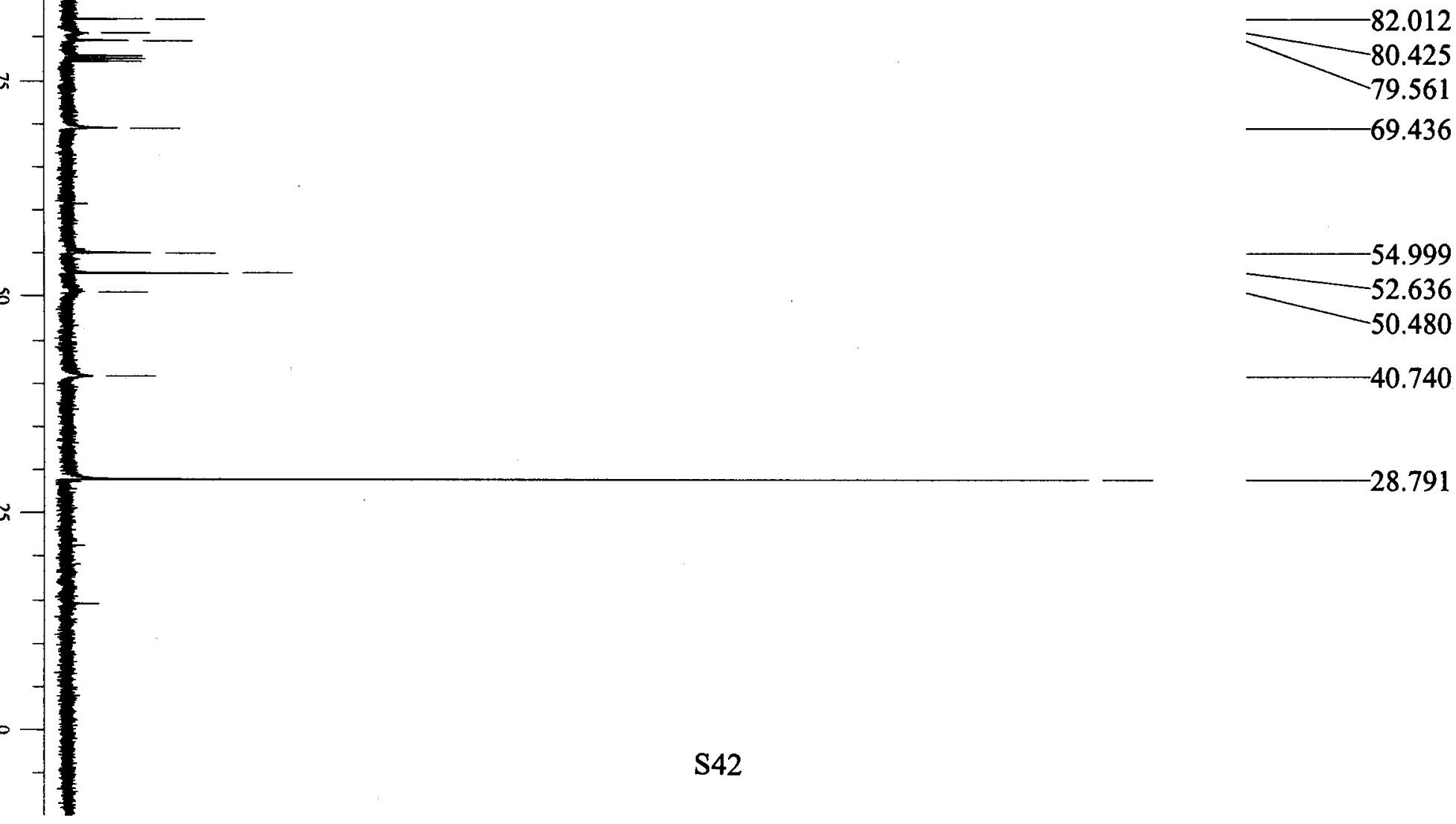

$-40.740$

28.791 


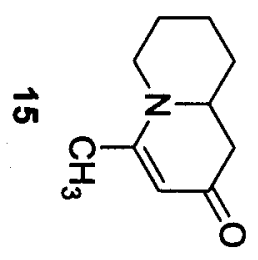




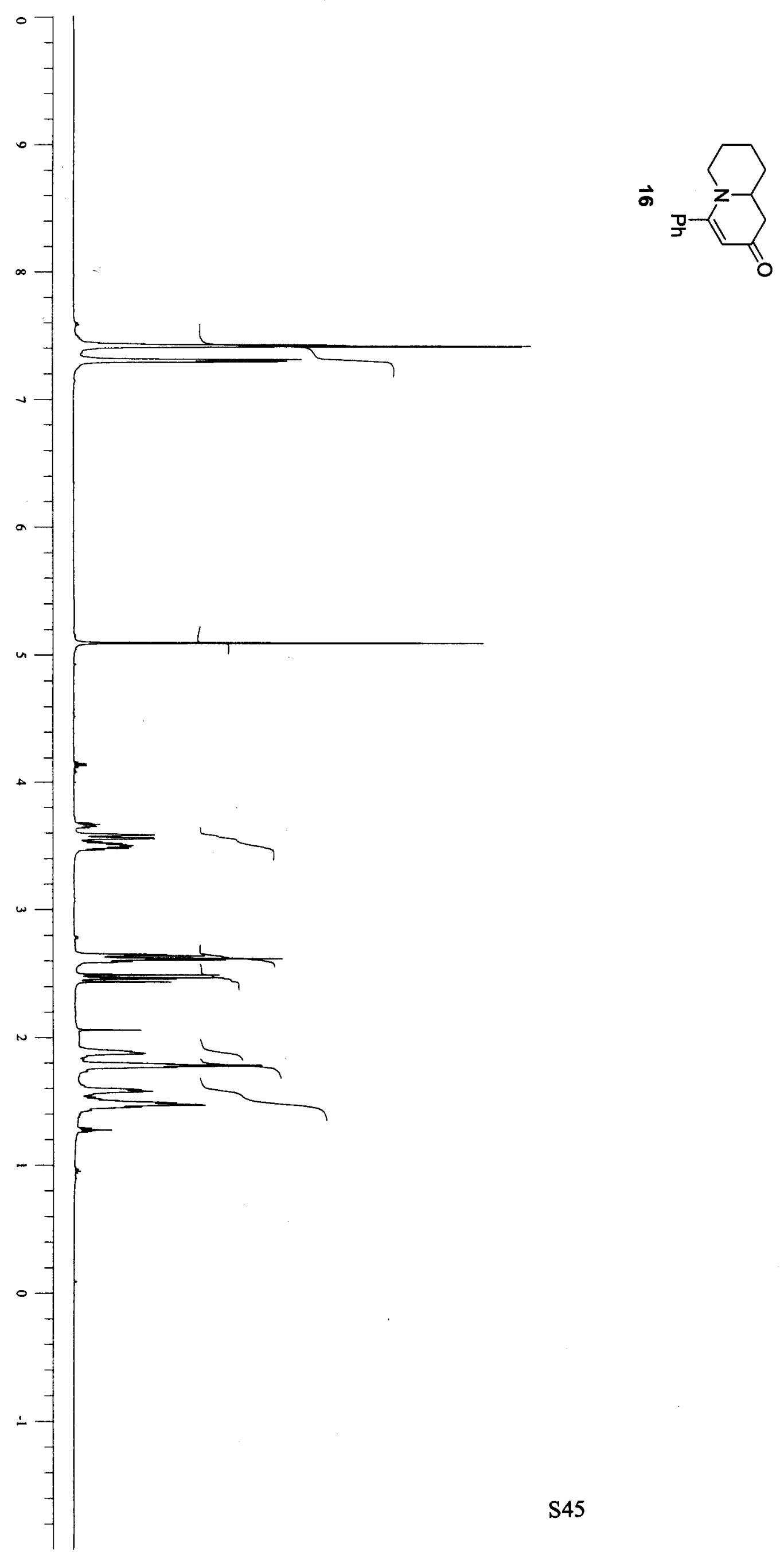



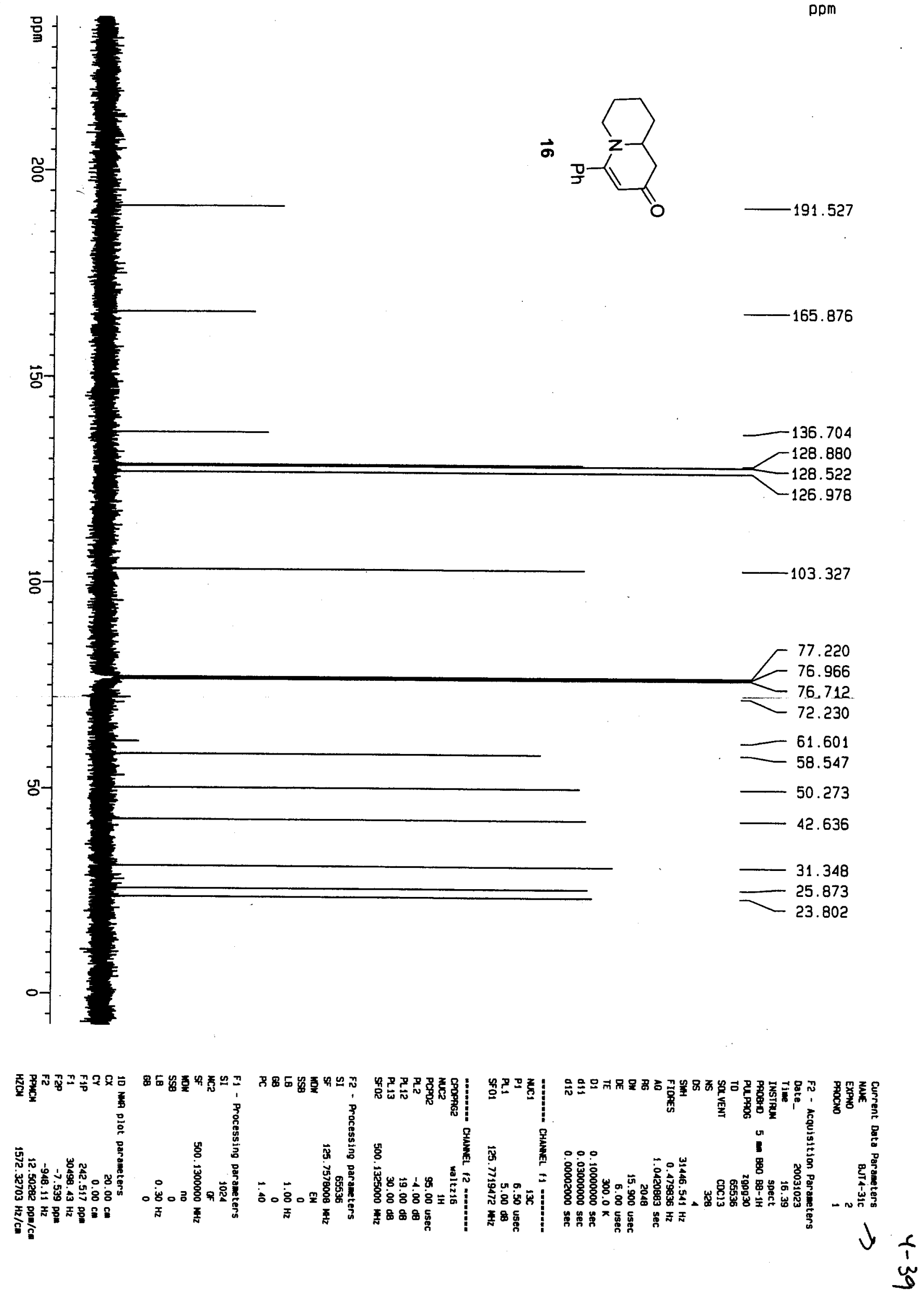


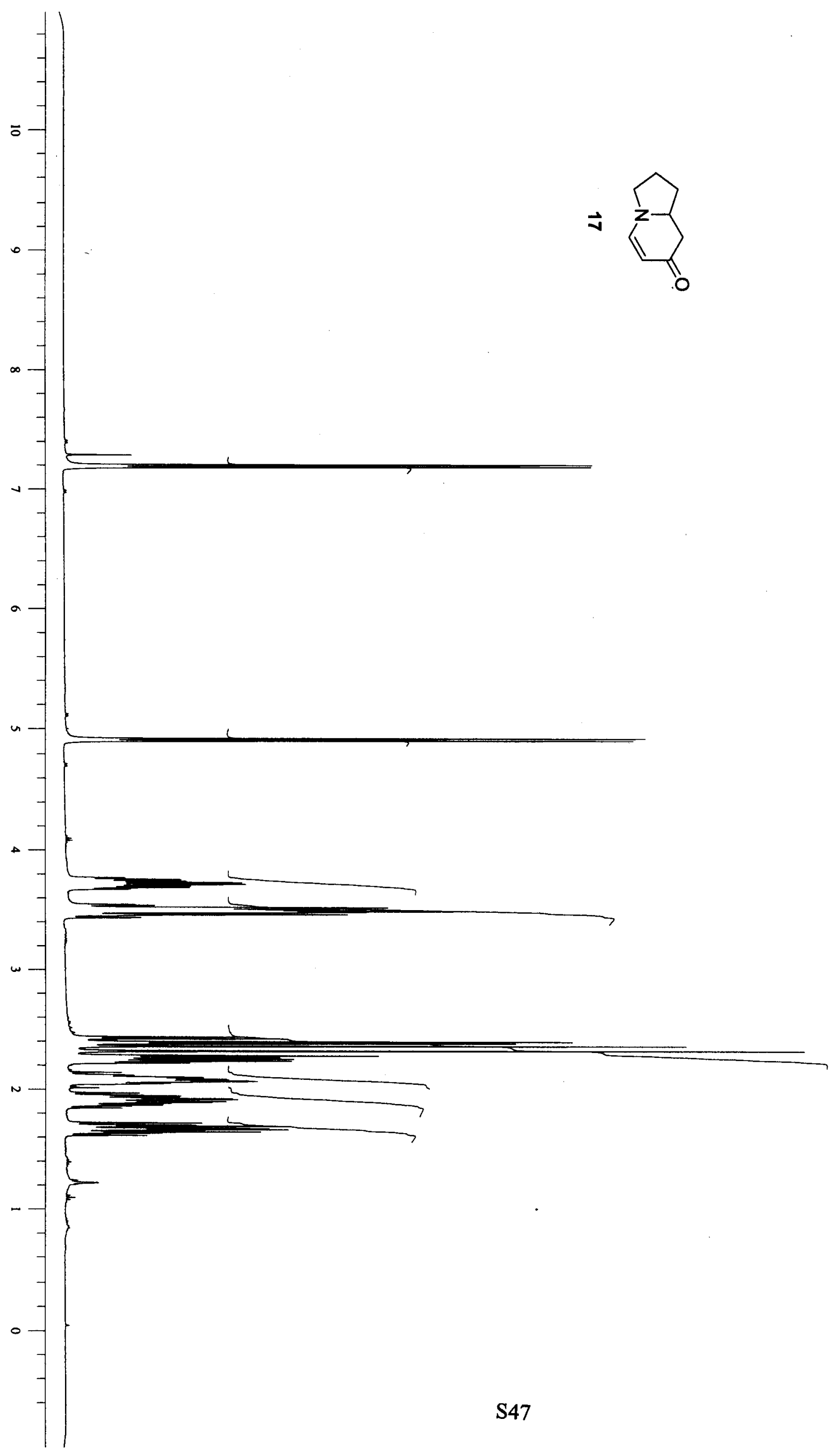




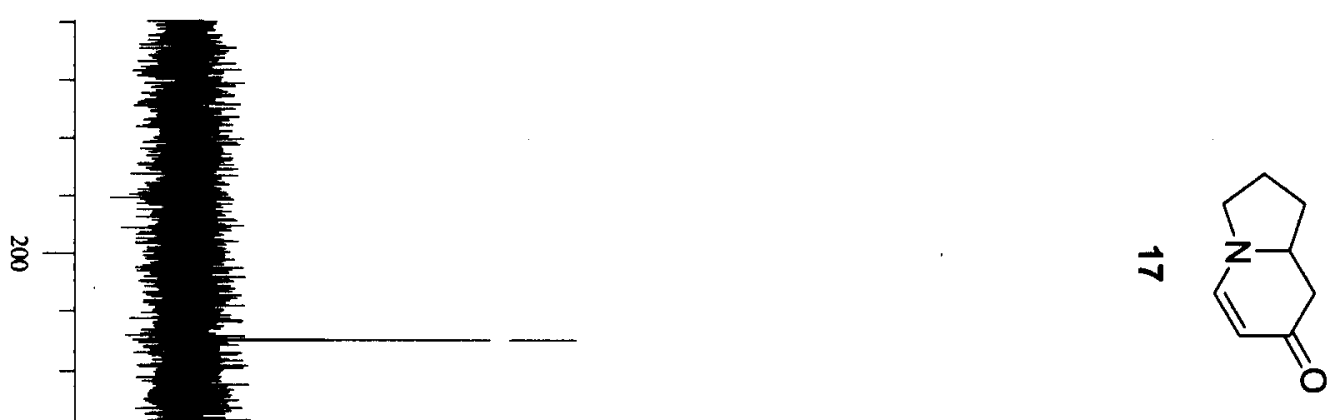




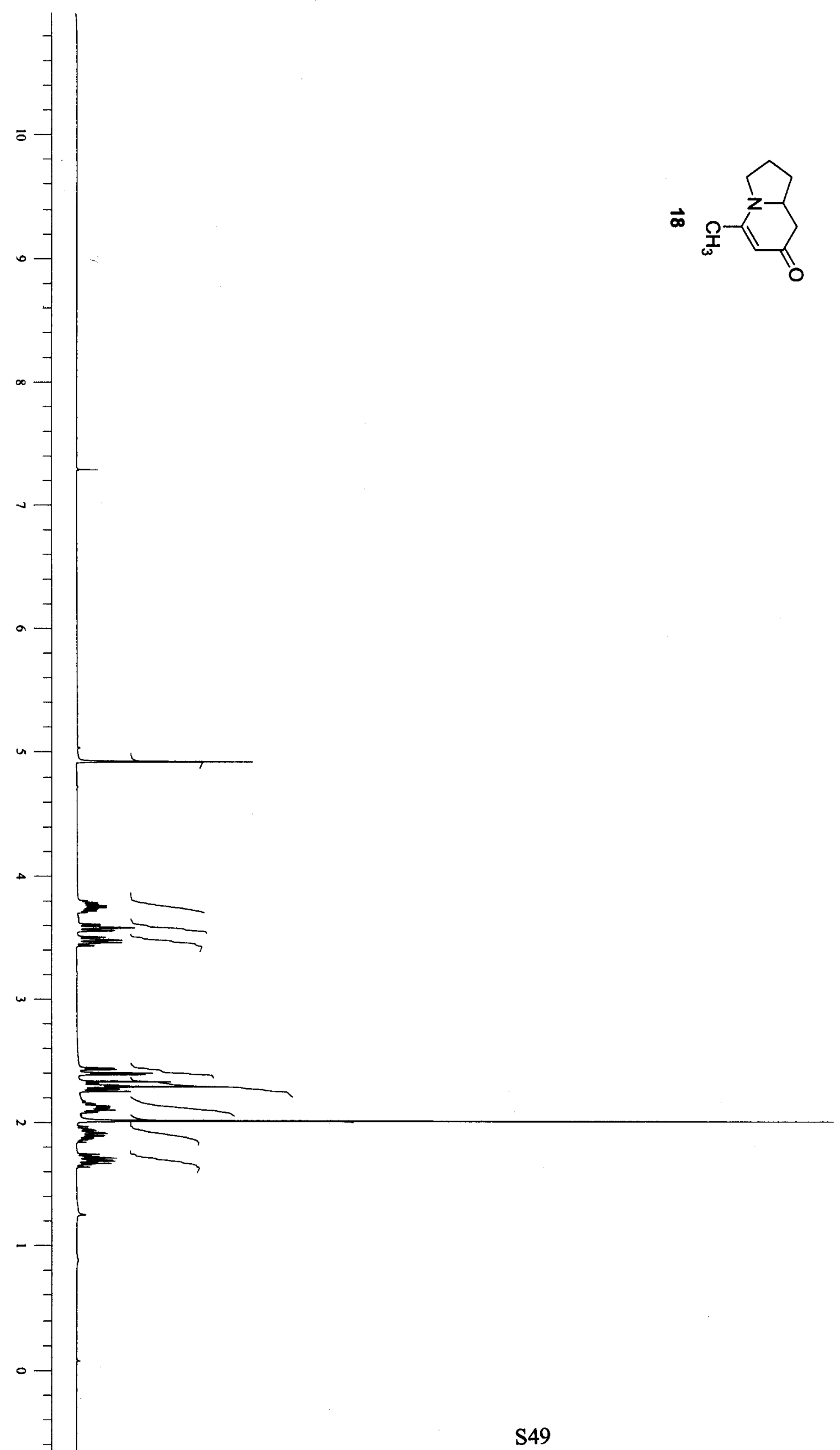




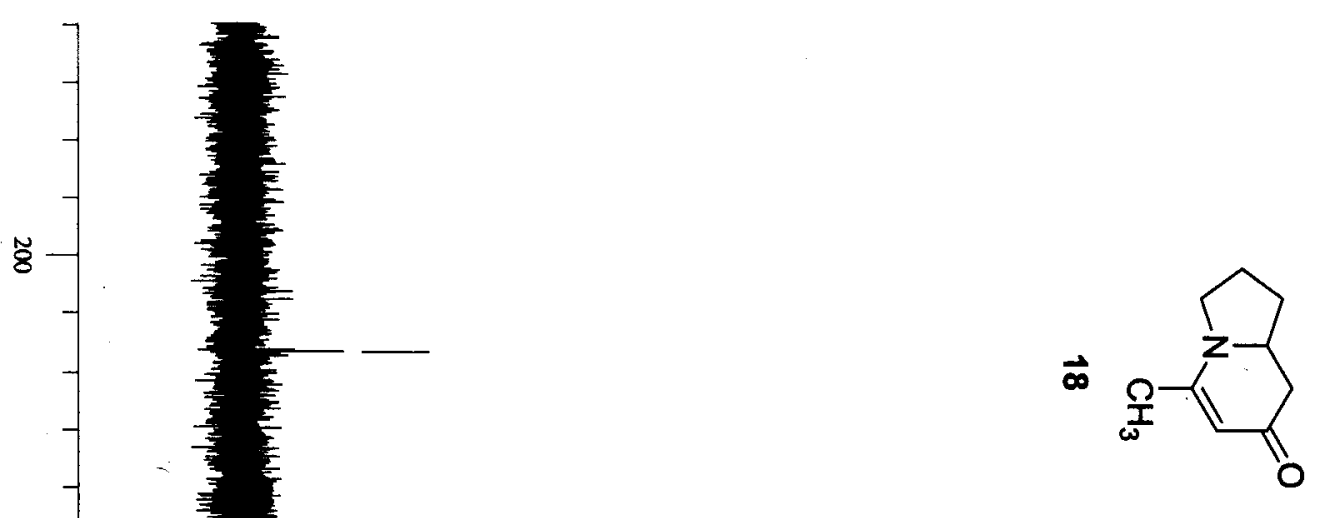

$-161.176$

$-98.414$

$-59.313$

47.332

41.661

$-33.109$

$-24.065$

20.892 


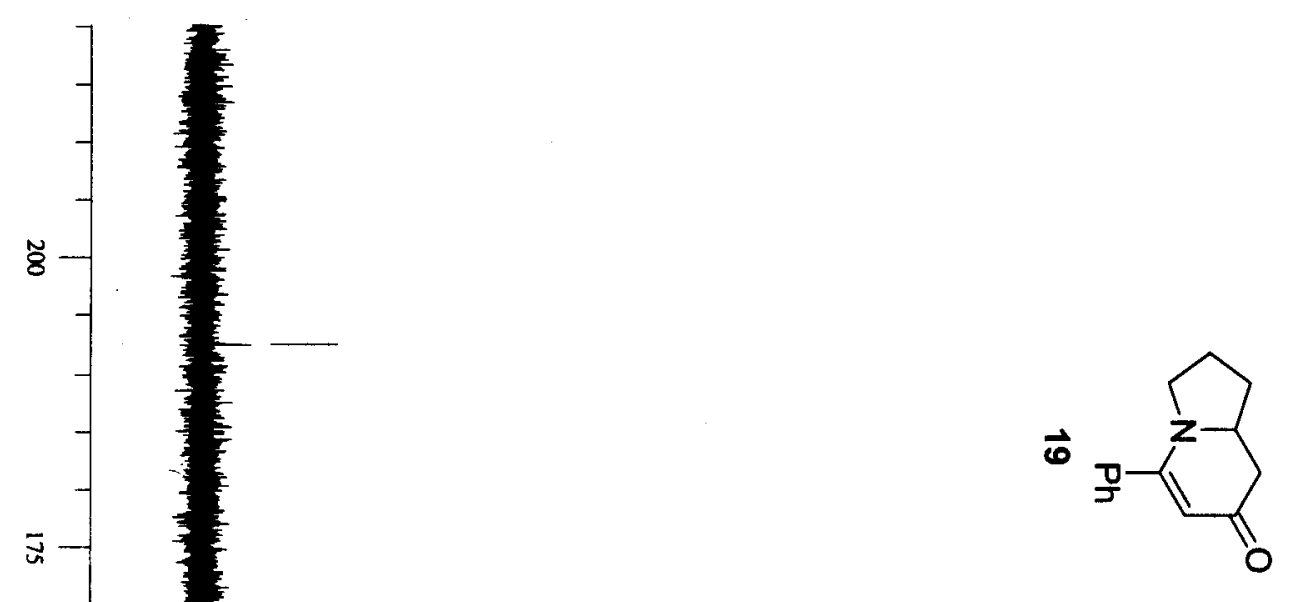

$-163.324$
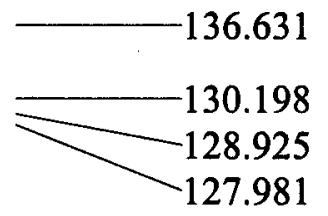

100.415

$-59.347$

$-49.686$

41.864

$-32.386$

$$
\tilde{n}
$$

$$
\text { . }
$$



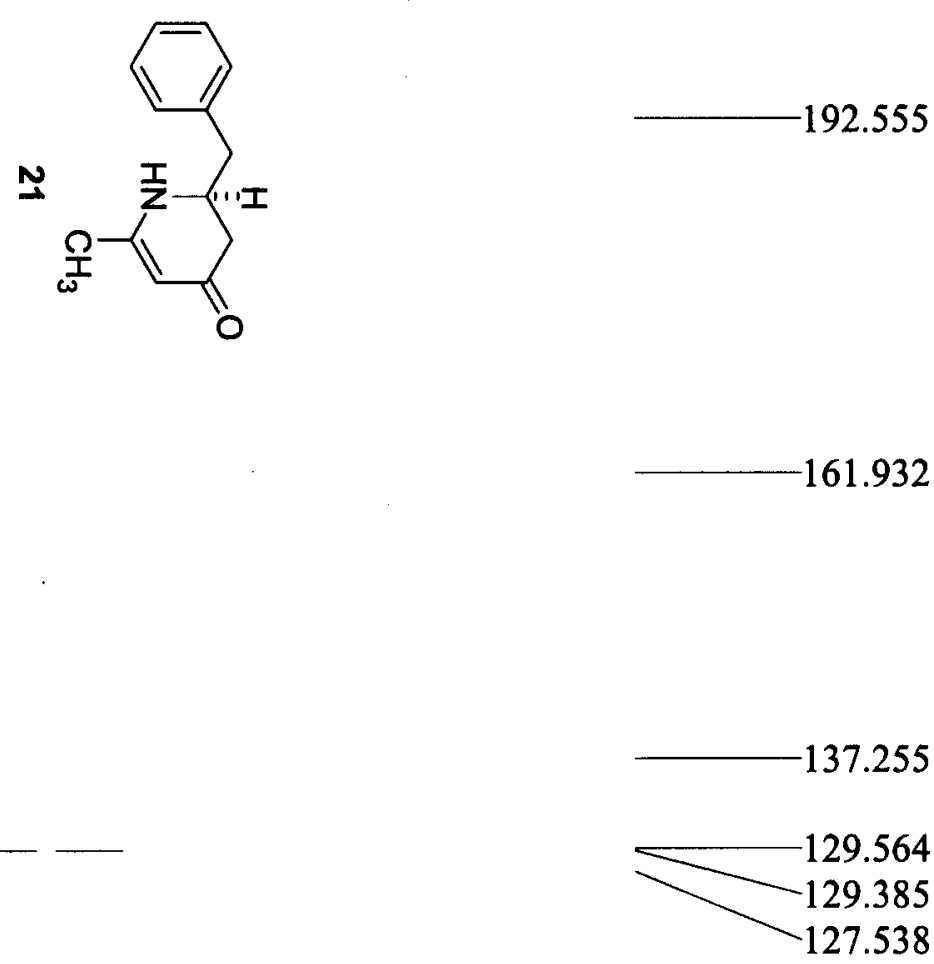

$-100.050$

54.712

sis
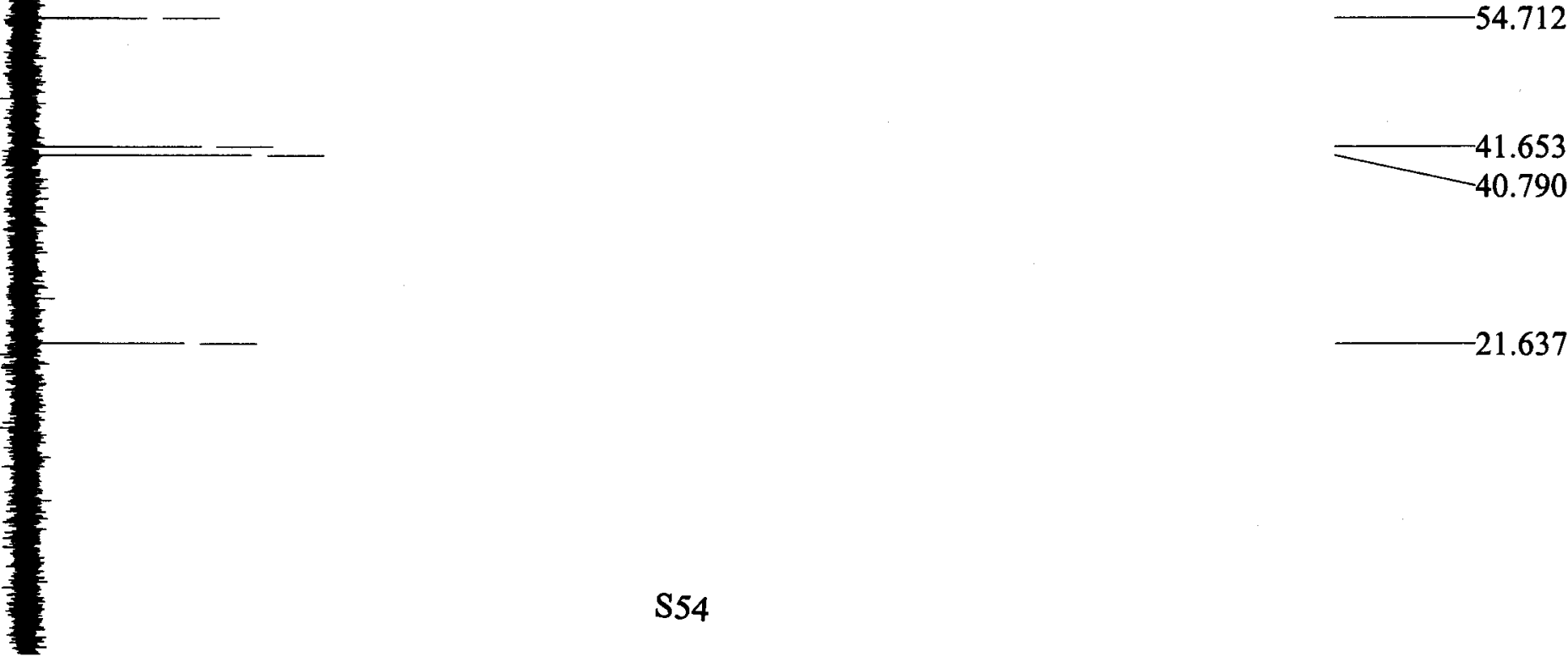

21.637 


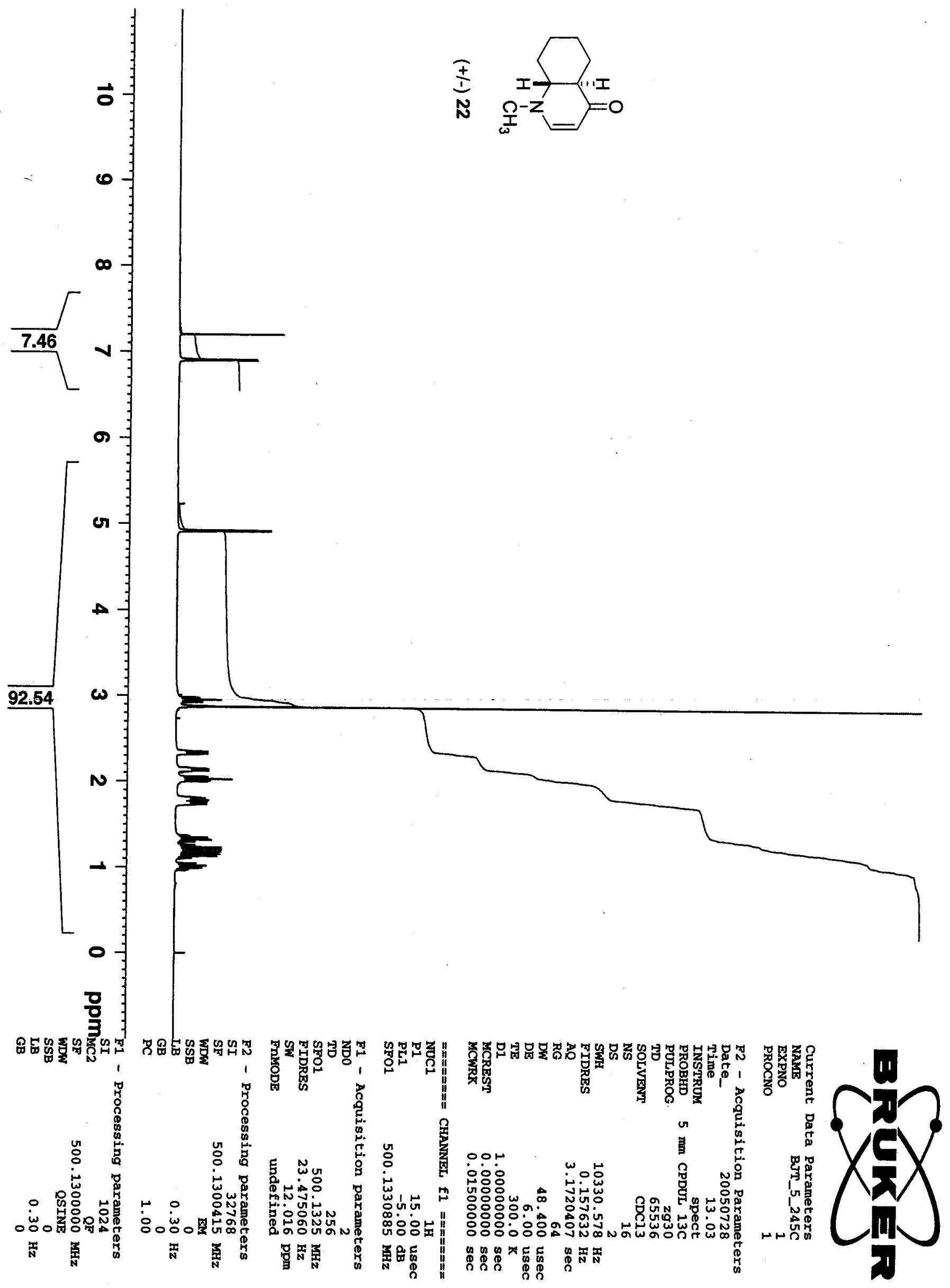



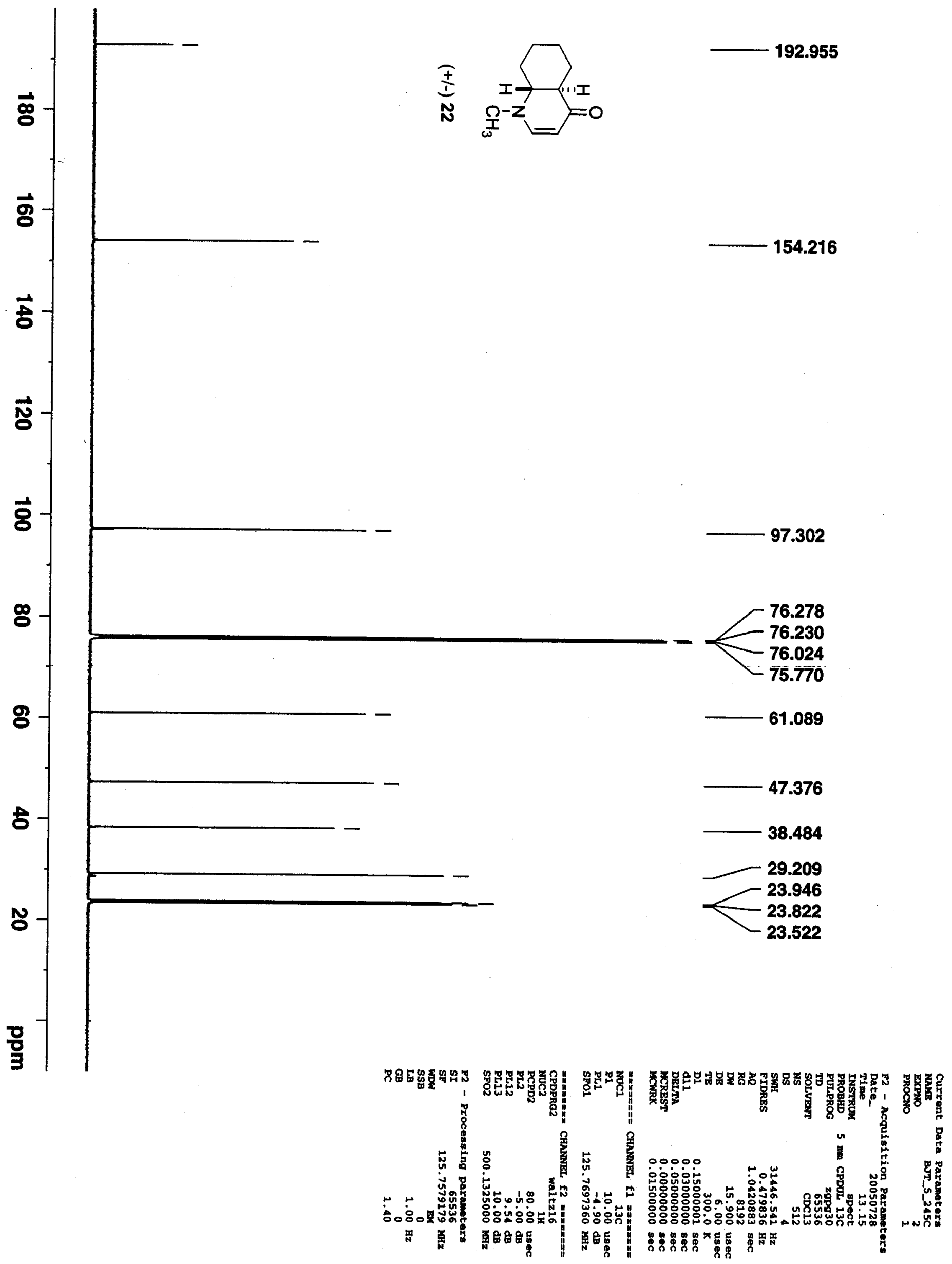

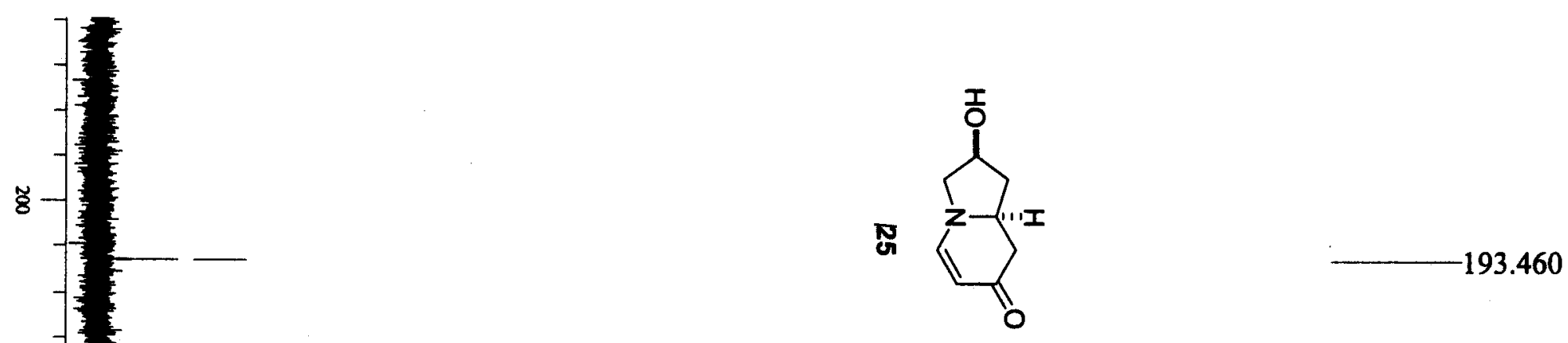

153.262

$-95.878$

69.878
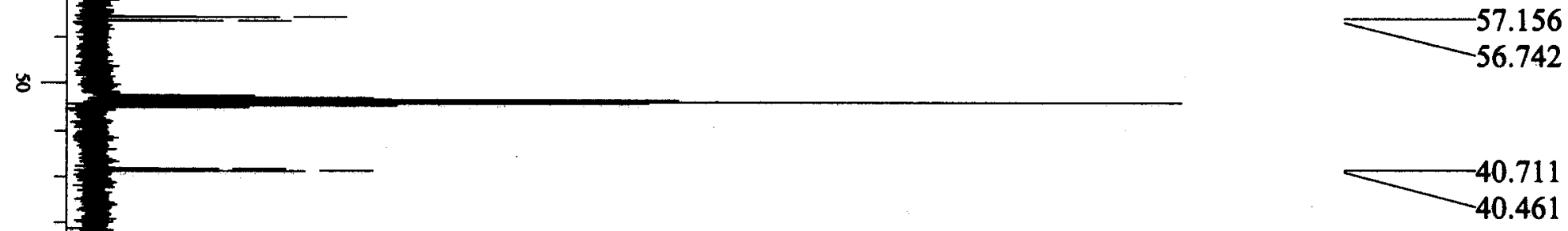

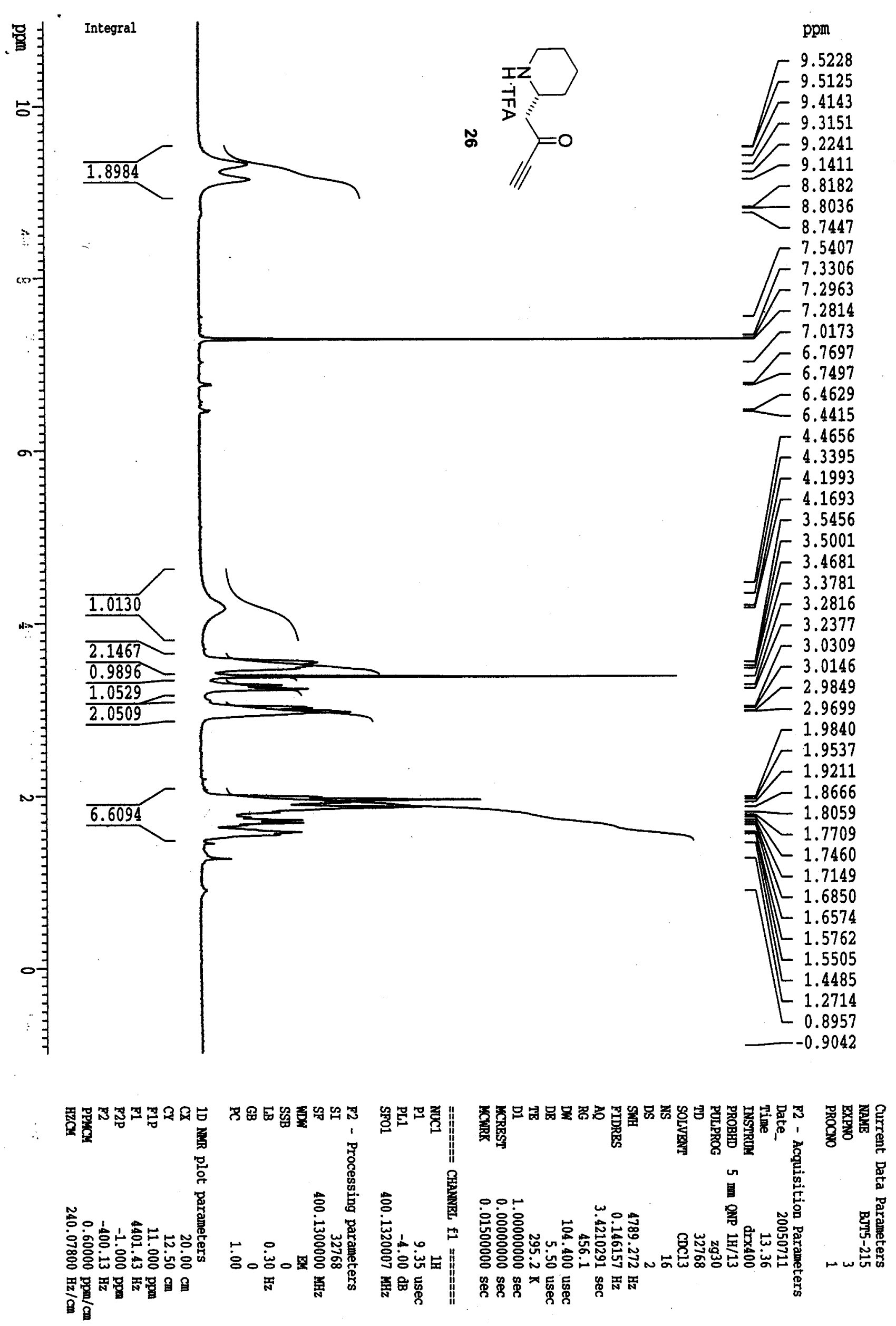

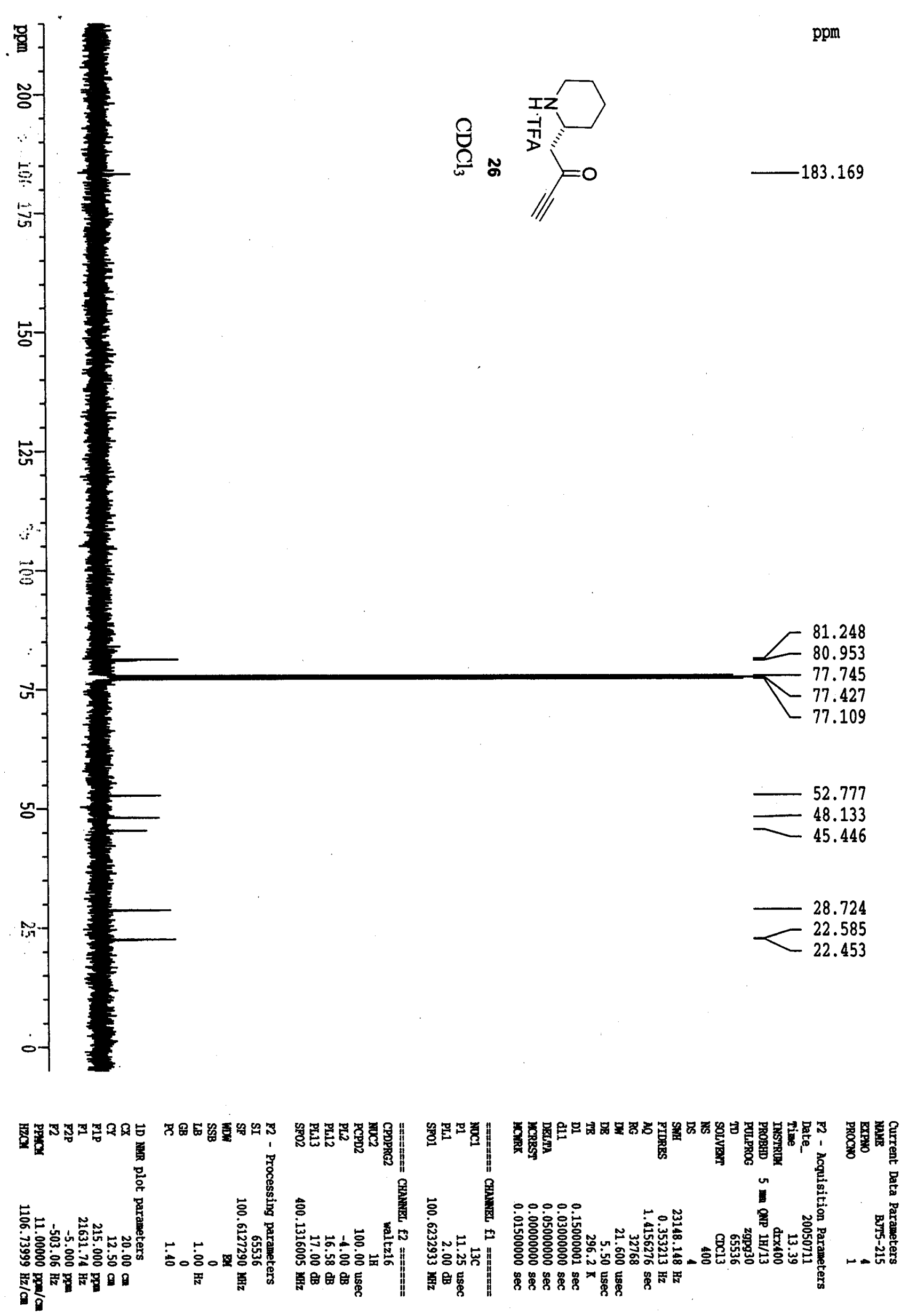


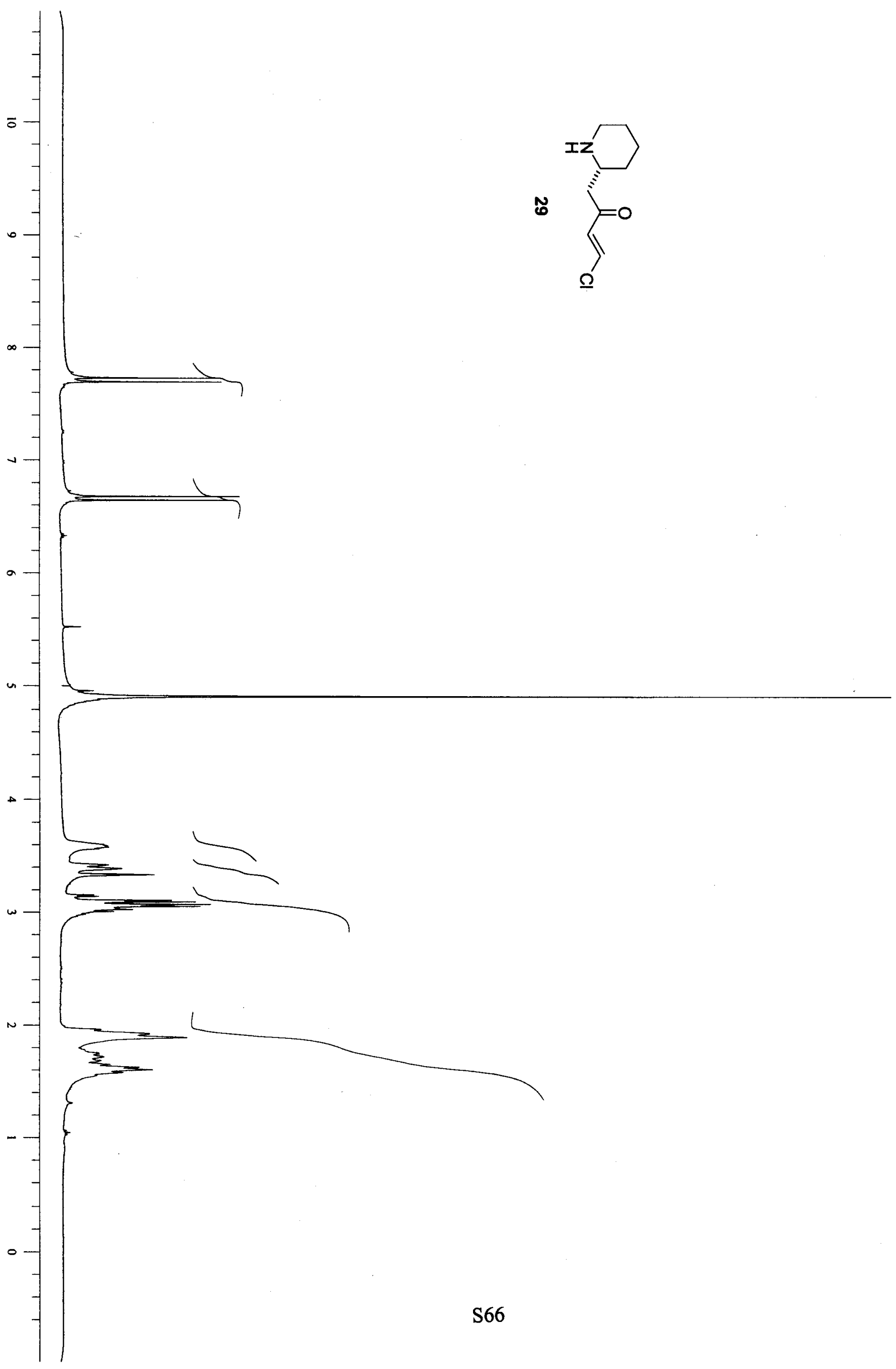




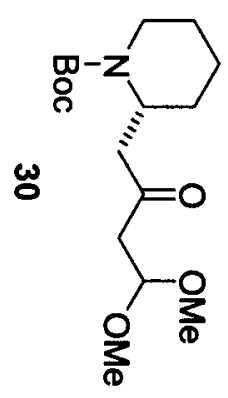

$-155.158$

$-102.113$

$-80.018$
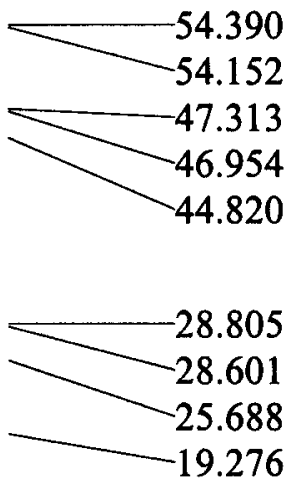